OPEN ACCESS

Edited by:

Sayeed Ahmad,

Jamia Hamdard University, India

Reviewed by:

Mohd Shahid,

Chicago State University,

United States

Washim Khan,

University of Mississippi, United States

*Correspondence:

Xiangning Cui

cuixiangning@126.com

Yingdong Lu

yingdonglu1988@126.com

${ }^{\dagger}$ These authors have contributed equally to this work

Specialty section:

This article was submitted to Ethnopharmacology,

a section of the journal

Frontiers in Pharmacology

Received: 14 July 2020 Accepted: 15 September 2020 Published: 02 November 2020

Citation:

Xin L, Gao J, Lin H, Qu Y, Shang C,

Wang Y, Lu Y and Cui X (2020)

Regulatory Mechanisms of Baicalin in Cardiovascular Diseases: A Review.

Front. Pharmacol. 11:583200. doi: 10.3389/fphar.2020.583200

\section{Regulatory Mechanisms of Baicalin in Cardiovascular Diseases: A Review}

\author{
Laiyun Xin ${ }^{1,2 t}$, Jialiang $\mathrm{Gao}^{2 \dagger}$, Hongchen $\mathrm{Lin}^{2}, \mathrm{Yi} \mathrm{Qu}^{2}$, Chang Shang ${ }^{2}$, Yuling Wang ${ }^{2}$, \\ Yingdong $\mathrm{Lu}^{2 \star}$ and Xiangning $\mathrm{Cu}^{2 *}$ \\ ${ }^{1}$ The First Clinical Medical College, Shandong University of Traditional Chinese Medicine, Jinan, China, ${ }^{2}$ Department of \\ Cardiology, Guang' anmen Hospital, China Academy of Chinese Medical Sciences, Beijing, China
}

Cardiovascular diseases (CVDs) is the leading cause of high morbidity and mortality worldwide, which emphasizes the urgent necessity to develop new pharmacotherapies. In eastern countries, traditional Chinese medicine Scutellaria baicalensis Georgi has been used clinically for thousands of years. Baicalin is one of the main active ingredients extracted from Chinese herbal medicine S. baicalensis. Emerging evidence has established that baicalin improves chronic inflammation, immune imbalance, disturbances in lipid metabolism, apoptosis and oxidative stress. Thereby it offers beneficial roles against the initiation and progression of CVDs such as atherosclerosis, hypertension, myocardial infarction and reperfusion, and heart failure. In this review, we summarize the pharmacological features and relevant mechanisms by which baicalin regulates CVDs in the hope to reveal its application for CVDs prevention and/or therapy.

Keywords: baicalin, cardiovascular diseases, inflammation, oxygen reactive species, apoptosis, immunomodulatory

\section{INTRODUCTION}

Cardiovascular diseases (CVDs) have become the leading cause of disability and death on a global scale (Timmis et al., 2018; Virani et al., 2020). CVDs lead to nearly one in three deaths in developed countries and one of every four deaths in developing countries according to epidemiological studies (Heidenreich et al., 2011; Zhao D. et al., 2019). Given the unmet needs in CVD control from Western medicine (Aggarwal et al., 2017), a complementary and alternative approach for treatment of CVD is needed. Traditional Chinese medicine (TCM), owning a history of 2,000 years, has drawn growing attention from the cardiovascular research community due to its their "multiple targets and multiple channels". Importantly, according to current guidelines, Chinese patients with coronary heart disease tend to use less Western medicine while applying more types of TCM (Guo et al., 2013). Moreover, TCM is increasingly welcomed in many developed countries, including the United States and Australia.

Baicalin is a monomeric flavonoid compound extracted from the root of Scutellaria baicalensis Georgi (SBG), a species of flowering plant in the Lamiaceae family (Chen et al., 1994). Baicalin is found in the root (10.11\%), which is the main medicinal part of SBG (Figure 1) (Shang et al., 2010; Dinda et al., 2017). SBG is an important ingredient in Xiaochaihu preparations, Gegenqinlian decocation and Sho-saiko-to preparations whose extracts include a key component of baicalin (Zhang, 1974; Srinivas, 2010; Zhao et al., 2016). Previous studies have high-lighted the extensive 


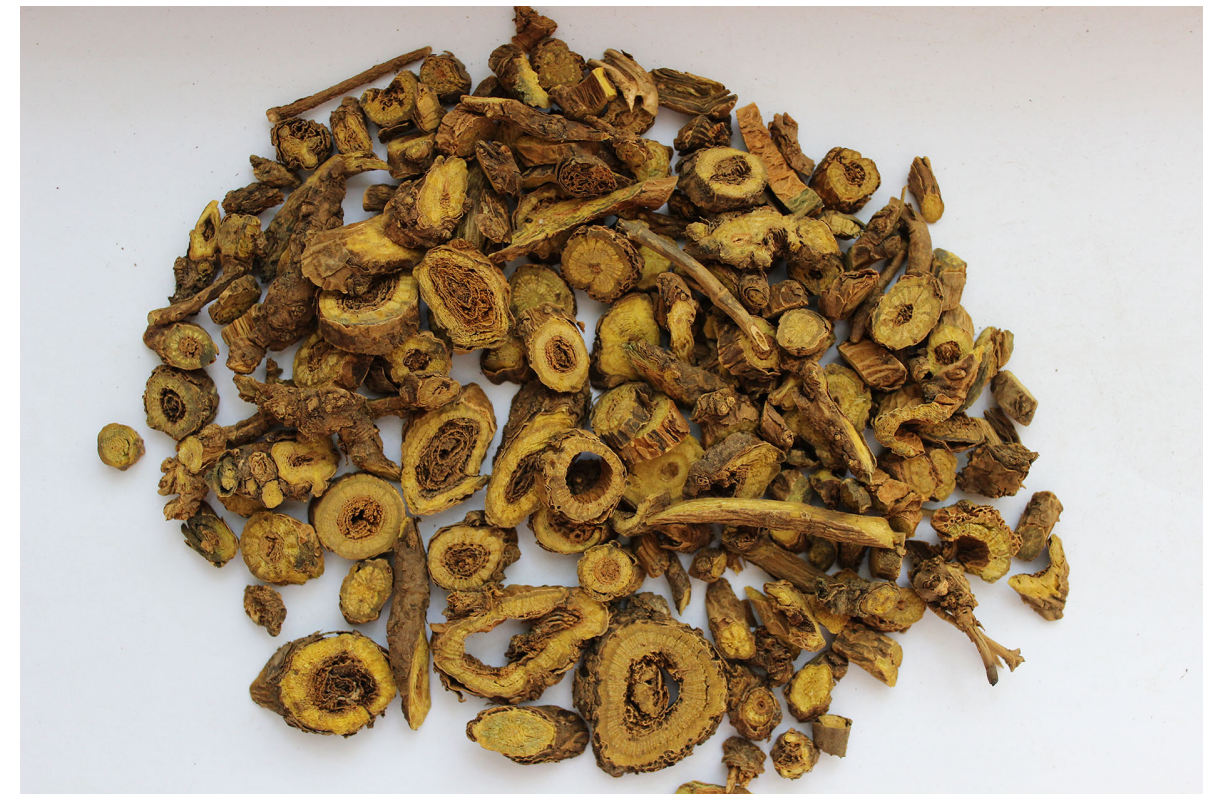

FIGURE 1 | The medicinal part of Scutellaria baicalensis Georgi and the main extraction source of baicalin.

pharmacological properties of baicalin for treating CVDs such as atherosclerosis (AS), hypertension, and ischemic heart disease. Considering its therapeutic mechanism may be associated with hypolipidemic, anti-inflammatory, anti-oxidant, anti-apoptosis properties (Chen et al., 2015; He et al., 2016; Dai et al., 2017) and considering its pharmacological properties and therapeutic potentials in the progression of CVDs, herein, we summarize and generalize the regulatory mechanisms involving baicalin in CVDs pathogenesis with a view to provide a reference for the development of new drugs for the treatment of CVDs.

\section{PHARMACOLOGICAL FEATURES OF BAICALIN}

\section{Bioactive Components in SBG}

Despite the popular clinical use of SBG, its active ingredients and the molecular mechanisms have not been fully clarified in detail to date. It has been reported that extracts of SBG and its major chemical constituents possess anti-viral, anti-tumor, antioxidant, anti-inflammatory, and neuroprotective activities (Wang Z. L. et al., 2018). With the deepening of understanding, increasing attention paid by the cardiovascular research community is focused on the bioactive chemical monomers comprising SBG that are responsible for its pharmacological activities. To date, many chemical constituents such as flavonoids, volatile oils, terpenoids, polysaccharides, steroids and amides in SBG have been isolated and identified. Flavonoids and their glycosides are considered to be characteristic components of SBG. According to the literature research, the most representative ingredients are baicalin, baicalein, wogonoside and wogonin (Li C. et al., 2011;
Zhao T. et al., 2019). Baicalin has poor solubility in water and lipid. In terms of the involvement of these chemicals in the development of agents for the treatment of CVDs, baicalin $\left(\mathrm{C}_{21} \mathrm{H}_{18} \mathrm{O}_{11}\right)$ is not only the first reported and the most abundant component but it also the component with greatest potential and value of those investigated (Figure 2) (Azimova and Vinogradova, 2013; Zhao T. et al., 2019).

\section{Pharmacokinetics of Baicalin}

Based on some advanced detection methods, pharmacokinetic investigations involving baicalin have been broadly carried out in the past few years. Absorption studies indicate that baicalin is poorly absorbed from the gastrointestinal tract in its native form and must be transformed into its aglycon baicalein through the hydrolysis by the intestinal bacteria, then baicalein can be restored to baicalin by UDP-glucuronosyltransferase (UGT) in the systemic circulation (Lu et al., 2007; Noh et al., 2016; Zhang et al., 2017). Since baicalein is optimally absorbed in all segments of the gastrointestinal tract, the key step in the absorption process of baicalin is the conversion of baicalin to baicalein (Huang et al., 2019). Furthermore, baicalin showed bimodal or multiple peaks in its absorption profile (Huang et al., 2012; Zhao et al., 2014; Zhang J. et al., 2016). The first peak was reported to occur within $45 \mathrm{~min}$ of ingestion, which was probably due to direct absorption, while the second peak occurred after 8-12 h [intravenously (i.v.)] or 12-24 h [orally (p.o.)] and was probably associated with the enterohepatic circulation (Lu et al., 2007; Huang et al., 2019). Next in terms its distribution, the binding rate of baicalin and plasma protein was found to range from 86$92 \%$, and the high plasma protein-binding rate allows baicalin to be absorbed rapidly into the plasma (Tang et al., 2006). A tissue distribution study showed that baicalin was found to accumulate 


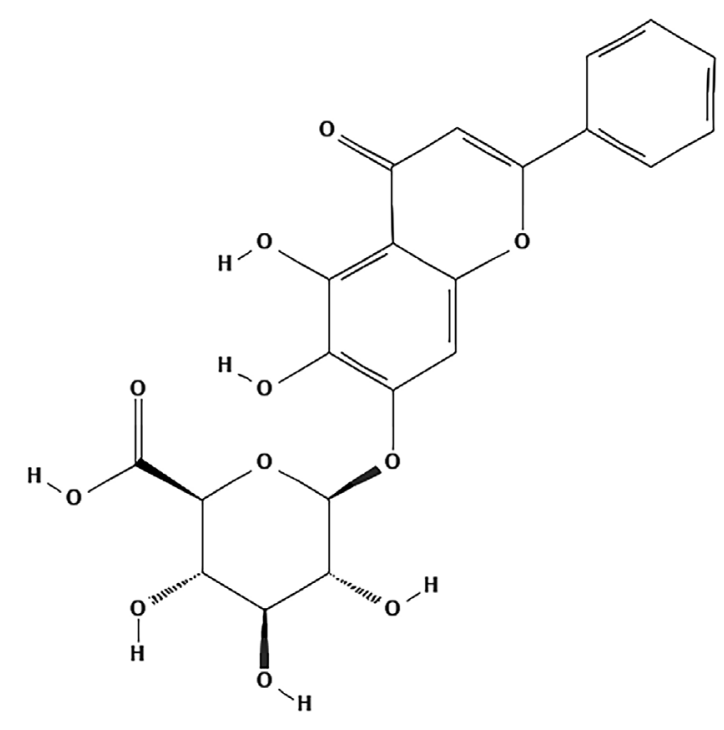

FIGURE 2 | The chemical structure of baicalin.

in various tissues and was highest in the kidneys after injection (Wei et al., 2016). Lastly, the metabolism and excretion of baicalin follows extensive metabolization by the liver and kidneys and is excreted in bile in the form of glucuronides. After oral administration of baicalin, the total cumulative amount of its glucuronides was determined to be about $54 \%$ of the dose (Abe et al., 1990). The major active metabolic sites are the hydroxyl groups on the A ring and 8- and 4'-positions of baicalin and several metabolic enzymes like $\beta$-glucuronidase, UGT, sulfatase and catechol-O-methyltransferases are also involved (Wang et al., 2012; Lu et al., 2012; Akao et al., 2013). Data have shown that the elimination half-life of baicalin is 0.1 till $4 \mathrm{~h}$ post dose (i.v) and $12.1 \mathrm{~h}$ (oral administration) (Xing et al., 2005). Therefore, its insolubility in water and lipid, extensive metabolism and high biliary excretion may contribute to the low bioavailability and short half-life of baicalin. Moreover, pathological conditions may alter the functions of many enzymes and transporters in vivo, thus baicalin may exhibit different pharmacokinetic properties under different pathological conditions (Xing et al., 2005; Huang et al., 2019). For instance, baicalin plasma concentration was higher in type 2 diabetic rats than normal rats (Liu et al., 2010). In cerebral ischemia-reperfusion rats, absorption of baicalin was enhanced, whereas it decreased in febrile rats (Ma et al., 2012; Huo et al., 2017).

\section{Drug Delivery Systems}

As a result of the low bioavailability of baicalin, this seriously affected its clinical development, although many new delivery strategies have been designed and developed, including nanonization technology, phospholipid complexes, solid dispersion, inclusion complexes, and micelles ( $\mathrm{Li} \mathrm{B}$. et al., 2011; Li N. et al., 2011; Yue et al., 2013; Zhang H. et al., 2016; Li et al., 2017). When compared to baicalin alone, modified preparations can improve its dissolution and solubility, which in turn further improve its targeting ability and therapeutic efficacy. For example, the baicalin liposomes modified with folic acid and polyethylene glycol (PEG) can improve the cellular uptake rate and tumor targeting, as well as extend the retention time in the body (Chen et al., 2016). Baicalin liposomes are also known to strengthen oral bioavailability and tissue distribution (Wei et al., 2014). Nonetheless, to date, limited information is available relative to baicalin drug delivery systems for AS therapy. Given the potential clinical applicability of baicalin in the future, suitable vehicles loading baicalin for use in alleviating AS progression deserve further evaluation.

\section{MOLECULAR MECHANISMS AND THERAPEUTIC POTENTIAL OF BAICALIN IN AS, MYOCARDIAL ISCHEMIA- REPERFUSION INJURY, HYPERTENSION, AND HEART FAILURE}

\section{Atherosclerosis}

AS is the primary pathological basis of CVDs, which could lead to dramatic clinical events, such as unstable angina or myocardial infarction (MI) (Reiner et al., 2011). The underlying pathophysiological mechanisms of AS involve endothelial dysfunction, lipid deposition, oxidative stress damage, immune inflammatory response, and platelet migration and aggregation (Packard et al., 2009; Weber and Noels, 2011; Gargiulo et al., 2016; Negre-Salvayre et al., 2017; Wolf and Ley, 2019). Accumulating studies indicate that baicalin can exert protective effects against AS by targeting these proatherogenic processes (Figure 3).

\section{Hypolipidemic Effects and Inhibition of Foam Cell Formation}

AS is a lipid-driven inflammatory disease of the arterial intima (Bäck et al., 2019). Lipid retention in the endothelium exacerbates the permeability of the vascular wall to promote the oxidation and deposition of lipoproteins which trigger the atherosclerotic cascade (Olofsson and Borèn, 2005). Highly effective lipid-lowing drugs are widely used in AS. A clinical trial has shown that baicalin could improve the state of blood lipid disorders and showed better improvements in patients with coronary artery disease, which was associated with decreasing the levels of serum total cholesterol (TC), triacylglycerol (TG), low-density lipoprotein cholesterol (LDL-C) levels and apolipoproteins. Furthermore, in ApoE models, baicalin decreased the lipid profile of TC, TG and LDL-C and ameliorated the progression of AS in a dose-dependent manner (Lee et al., 2009; Hang et al., 2018). Several biological processes associated with baicalin are involved in lipid regulation activities. In terms of the molecular mechanisms involved in baicalin-modulated lipid regulation activities, an vitro study of 3T3-L1 preadipocytes determined that baicalin downregulated the expression levels of major transcription factors of 


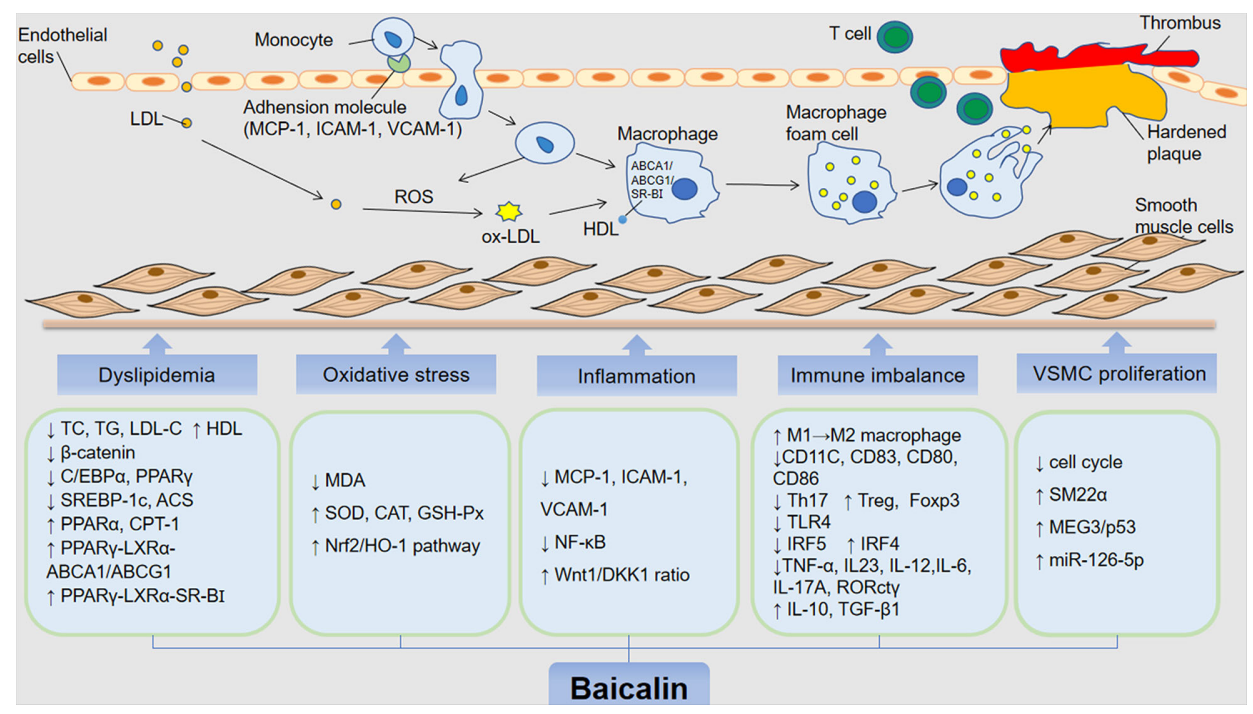

FIGURE 3 | The anti-atherosclerotic effects by which baicalin alleviates the development of AS. The role of baicalin in inhibiting atherosclerosis includes hypolipidemic effects and inhibition of foam cell formation, inhibition of oxidative stress, mitigation of inflammatory response, immunomodulatory, suppression of VSMC proliferation and migration. low density lipoprotein (LDL); oxidized LDL (oxLDL); oxygen reactive species (ROS); high-density lipoprotein (HDL); total cholestero (TC); triacylglycerol (TG); low-density lipoprotein cholesterol (LDL-C); CCAAT/enhancer bindingproteins $\alpha(\mathrm{C} / \mathrm{EBP} \alpha)$; peroxisome proliferator-activated receptor $\gamma(\mathrm{PPAR} \gamma)$; liver receptor X (LXR $\alpha)$; ATP binding cassette (ABC) transporter A1/G1 (ABCA1/G1); scavenger receptor BI (SR-BI); sterol regulatory element-binding protein 1c (SREBP-1c); Acyl-CoA synthase (ACS); peroxisome proliferator-activated receptor $\alpha$ (PPAR $\alpha$ ); carnitine palmitoyltransferase 1 (CPT-1); malondialdehyde (MDA); superoxide dismutase (SOD); catalase (CAT); glutathione peroxidase (GSH-Px); nuclear factor E2-associated factor 2 (Nrf2); heme oxygenase-1 (HO-1); monocyte chemoattractant protein 1 (MCP-1); intercellular adhesion molecule 1 (ICAM-1); vascular adhesion molecule 1 NCAM-1); nuclear factor kappa B (NF-кB); dickkopf-related protein-1 (DKK 1); interleukin 10/12/23/6/17A (IL-10); interferon regulatory factor 4 and 5 (IRF4/5); tumor necrosis factor (TNF)- $\alpha$; transforming growth factor- $\beta 1$ (TGF- $\beta 1$ ); toll-like receptor (TLR4); smooth muscle (SM) $22 \alpha$; factor forkhead box protein P3 (Foxp3); maternally expressed gene 3/p53 (MEG3/p53).

adipogenesis including CCAAT/enhancer binding proteins $(\mathrm{C} / \mathrm{EBP} \alpha)$ and peroxisome proliferator-activated receptor (PPAR) $\gamma$ (Lee et al., 2009). $\beta$-Catenin, upstream of PPAR $\gamma$ and the $\mathrm{C} / \mathrm{EBP} \alpha$ regulator, has a negative relationship with adipogenesis. Baicalin significantly attenuated $\beta$-catenin expression in an in vitro study (Lee et al., 2010). Furthermore, another study indicated that baicalin could lower TG levels by favoring lipid oxidation by blocking the expression of sterol regulatory element-binding protein 1c (SREBP-1c) and AcylCoA synthase (ACS) apart from increasing the expression of lipolysis-related proteins such including PPAR $\alpha$ and carnitine palmitoyltransferase 1 (CPT-1) (Wu et al., 2018).

The formation of foam cells occurs in the early phases of AS. The scavenger receptors, such as CD36 and scavenger receptors class A (SR-A) expressed in macrophages combines with oxidized LDL (oxLDL), which is efficiently decomposed and metabolized by lysosomes into free cholesterol and is stored in the cytosol (Collot-Teixeira et al., 2007; Moore and Tabas, 2011; $\mathrm{Yu}$ et al., 2013). The accelerated accumulation of cholesterol induces the formation of foam cells. In contrast, cholesterol efflux, by which cholesterol-loaded macrophages within the vessel wall secrete cholesterol outside cells, is understood to be a major process in repressing macrophage conversion (Ohashi et al., 2005). The main transporters of cholesterol efflux include the adenosine triphosphate binding cassette transporters ABCA1/ABCG1 and scavenger receptor class B type I (SR-BI)
(Yu et al., 2013). In the New Zealand rabbit AS model, baicalin significantly enhances the expression of ABCA1 and ABCG1 (He et al., 2016). PPAR $\gamma$ is part of a metabolic cascade highly expressed in macrophage that enhances cholesterol efflux, and liver receptor X (LXR $\alpha)$ directly increases the expression of ABCA1 (Chawla et al., 2001). One study found that baicalin inhibited the accumulation of cholesterol and delayed the transformation of macrophages into foam cells, which was the result of the upregulation of the PPAR $\gamma$-LXR $\alpha$-ABCA1/ABCG1 pathway. However, the effects of baicalin on the expression of SR-A and CD36 were not obvious (He et al., 2016). As the highdensity lipoprotein (HDL) receptor, SR-BI has been identified as a critical role in promoting cholesterol efflux in macrophages $(\mathrm{Gu}$ et al., 2000; Trigatti et al., 2004). In a THP-1 macrophage study, researchers concluded that baicalin induced cholesterol efflux via the PPAR $\gamma$-LXR $\alpha$-SR-BI pathway (Yu et al., 2016). Furthermore, $\mathrm{HDL}$ is reported to be responsible for the reverse transport of cholesterol from the peripheral tissues to the liver for reuse or for the final elimination via excretion into the bile. Baicalin is capable of up-regulating the plasma levels of HDL, which implies that increasing HDL might be a mechanism of action for baicalin to expedite cholesterol ejection from macrophages and weaken their conversion (Hovingh et al., 2003; Xi et al., 2015). Taken together, it is rational to propose that baicalin might attenuate AS partly by modulating lipid metabolism and foam cells formation. 


\section{Inhibition of Oxidative Stress}

The imbalance between the overproduction of oxygen reactive species (ROS) in cells and tissues and the ability of a biological system to remove ROS products is called oxidative stress (Peluso et al., 2012; Pizzino et al., 2017). Under pathological conditions, oxidative stress exacerbates the AS. Plasma LDL maybe trapped and accumulate in injured vascular endothelium and be oxidized by ROS to ox-LDL. In addition, the recruited monocytes and migrated lymphocytes can also release large amounts of ROS and which favor the synergism of early atherosclerotic lesions. Moreover, ROS can promote vascular smooth muscle cells (VSMCs) proliferation and collagen deposition thereby leading to the development of an atheromatous plaque in the final (Tousoulis et al., 2015; Kattoor et al., 2017). Recently, considerable data has revealed that baicalin could on one hand attenuate AS by decreasing oxidative stress products and on the other hand strengthen the anti-oxidative system.

Firstly, baicalin may directly or indirectly reduce oxidative stress products. Malondialdehyde (MDA) is a decomposition product of lipid hydroperoxides and can be used as an indicator of oxidative damage to cells and tissues (Bonnes-Taourel et al., 1992). Baicalin was shown to effectively relieved the oxidative stress by abrogating the upregulation of MDA in ApoE-/- mice (Wu et al., 2018). Secondly, in terms of the effects of baicalin on the antioxidative system, the antioxidant enzymes in the vascular wall are not only important radical superoxide scavengers that protect cells from oxidative damage, but are also important parts of the endogenous antioxidant defense system (Olsvik et al., 2005; Ku et al., 2009; Hassan et al., 2014; Wu et al., 2018). The experiment designed by $\mathrm{Wu}$ suggested that baicalin treatment significantly relieved the oxidative stress by up-regulating the activities of superoxide dismutase (SOD), catalase (CAT) and glutathione peroxidase (GSH-Px) in a dose-dependent manner compared to the AS model group (Wu et al., 2018). This suggests that baicalin can increase antioxidant enzyme activity and thus improve AS. Further, Nuclear factor E2-associated factor 2 (Nrf2), a basic leucine zipper transcription factor, regulates antioxidant proteins to prevent oxidative damage. As the "master regulator" of the antioxidant response, Nrf2 can modulate the expression of antioxidant enzymes such as heme oxygenase-1 (HO-1) (Loboda et al., 2016). It has been reported that $\mathrm{Nrf} 2 / \mathrm{HO}-1$ pathway exhibits protective roles in many ischemic disorders. Hypoxia stimulates the Nrf2/HO- 1 pathway activation in cardiomyocytes (Zeng et al., 2015). Baicalin treatment may enhance the Nrf2 and HO-1 expression and further activate the Nrf2/HO-1 pathway ( $\mathrm{Yu}$ et al., 2019). Taken together, the potent abilities in elevating antioxidant enzymes by baicalin might lead to the elimination of ROS and subsequently ameliorate oxidative stress-induced atheromatous plaque formation.

\section{Mitigation of the Inflammatory Response}

AS related inflammation mediated by chemokines, adhesion molecules, proinflammatory cytokines and inflammatory signaling pathways plays an important role in all stages of the atherosclerotic progression (Ross, 1999; Libby et al., 2011; Zhu et al., 2018). Injured endothelial cells activated by inflammatory factors stimulate the biosynthesis of monocyte chemoattractant protein 1 (MCP-1), intercellular adhesion molecule 1 (ICAM-1), vascular adhesion molecule 1 (VCAM-1), and pro-inflammatory cytokines that recruits circulating monocytes to the intima. Migrated monocytes differentiate into macrophages that contribute to inflammation and plaque development (Chistiakov et al., 2018). In an in vitro study, baicalin was found to decrease monocyte adhesion via reducing the expression of MCP-1, ICAM-1, and VCAM-1 in the presence of high glucose-induced human umbilical vein endothelial cells (HUVECs). Similarly, baicalin had been confirmed to markedly decrease the release of MCP-1, VCAM-1 and IL-6 in the kidney of apolipoprotein E (ApoE)' knockout (KO) mice (Liu et al., 2015). Further, baicalin inhibited the synthesis of neutrophil chemokines and blocked the biological activity of receptors by selectively binding chemokine receptors, thereby inhibiting the migration and aggregation of inflammatory cells and reducing the degree of AS (Li et al., 2000). Furthermore, in the development of AS-induced inflammation, inflammatory signaling pathways like nuclear factor kappa B (NF- $\mathrm{BB})$ and Wnt1/DKK1 also have a proatherogenetic impact from fatty streak formation to luminal occlusion. It has been reported that the activation of the $\mathrm{NF}-\mathrm{\kappa B}$ increases the production of inflammatory cytokines and chemokines that promote the progression of AS (Kutuk and Basaga, 2003). Thus, the inhibition of NF- $\kappa \mathrm{B}$ signaling has been shown to reduce the incidence of AS. Baicalin also suppressed the activation of $\mathrm{NF}-\kappa \mathrm{B}$ in human umbilical vein endothelial cells in previous studies (Kim et al., 2006; Ku and Bae, 2015). Another important mechanism of baicalin is mediated by the Wnt1/DKK1 signaling pathway. The Wnt1 signaling pathway is a highly conserved cellular communication system (Peifer and Polakis, 2000). Recent evidence suggests that Wnt signaling participates in inflammatory regulation and the pathogenesis of AS (Sen and Ghosh, 2008). Specifically, Wnt1 plays an anti-atherosclerotic role (Gherghe et al., 2011). Dickkopf-related protein-1 (DKK1), which interacts with Wnt, has recently been considered as a biomarker for AS (Niehrs, 2006; Ueland et al., 2009; Kim et al., 2011). Therefore, the intervention of the Wnt1/DKK1 pathway may represent a feedback approach to resist the progression of AS. A basic study designed by Wang et al. confirmed that baicalin exerted a regulatory effect on inflammation and prevented AS by promoting Wntl signally and inhibiting the expression of DKK1 through an elevated Wnt1/DKK1 ratio (Wang et al., 2016).

\section{Immunomodulatory Activity}

AS is a chronic, low-grade inflammatory response that attracts cells of the innate and adaptive immune systems to the atherosclerotic plaque (Libby, 2002). Experimental and clinical evidence has revealed that both innate and adaptive immunity play important roles in the onset and progression of AS (Wolf and Ley, 2019). The complex network of interactions among vascular components and immune cells regulate the balance 
between proatherogenic inflammatory and atheroprotective anti-inflammatory responses (Herrero-Fernandez et al., 2019).

The natural immune cells involved in AS mainly include mononuclear-macrophages and dendritic cells (DCs) (Shimada, 2009; Hansson and Hermansson, 2011; Ammirati et al., 2015). Circulating monocytes and resident macrophages are the earliest recruited to atherosclerotic plaques and the most abundant immune cells. Baicalin may prevent the proliferation of mononuclear cells and inhibit macrophage activation (Liu et al., 2008). Macrophages can be divided into classically activated pro-inflammatory (M1) and alternatively activated anti-inflammatory (M2) phenotypes (Tan et al., 2016). $\mathrm{M} 2_{\mathrm{C}}$ is one subtype of M2 macrophages and is mainly responsible for the phagocytosis of apoptotic cells (Zizzo et al., 2012). RAW264.7 macrophages in the M1 phenotype induced by lipopolysaccharide (LPS) could easily be switched to M2 phenotype after baicalin induction. Thus, baicalin may promote $\mathrm{M} 2{ }_{\mathrm{C}}$ macrophages polarization and increase the atherosclerotic plagues clearance (Lai et al., 2018). Previous study showed that baicalin could significantly increase the serum levels of interleukin (IL)-10 and transforming growth factor- $\beta 1$ (TGF- $\beta 1$ ) (Liao et al., 2014). Meanwhile, the upregulated expression of IL-10 might be involved in reducing tissue migration of neutrophils, boosting the formation of regulatory $\mathrm{T}$ cells and promoting phagocytic activity of $\mathrm{M} 2 \mathrm{c}$ (Van den Akker et al., 2013; Lai et al., 2018). Other studies had also shown that baicalin promoted M2 macrophage polarization in vitro by increasing interferon regulatory factor 4 (IRF4) protein expression and by decreasing M1 markers such as tumor necrosis factor $\alpha$ (TNF- $\alpha$ ), IL23, and IRF5 (Zhu W. et al., 2016). In an LPS-induced rat model, baicalin could also repress the toll-like receptor (TLR) 4 signaling pathway of the peripheral blood mononuclear cells (Ye et al., 2015). Furthermore, DCs, presenting oxLDL-derived antigens in atherosclerotic plaques and secondary lymphoid organs, could treat oxLDL in plaques and stimulate adaptive immune responses (Steinman et al., 1975; Steinman and Hemmi, 2006). Liu et al.'s research pointed out that baicalin inhibited the expression of the CD11c marker on DCs in aorta tissue and CD11C, CD83, CD80, and CD86 markers on DCs in the bone marrow. Thus, baicalin may have exert immune-regulatory effects and prevent the formation of atherosclerotic lesions by decreasing the DC numbers, and inhibiting DC maturation in bone marrow and infiltration into lesions (Liu et al., 2014). Baicalin can also affect DC-related inflammatory mediators, especially the expression of IL- 12 .

The adaptive immune system is also involved in AS progression. $\mathrm{CD}^{+} \mathrm{T}$ cells are the key regulatory cells for adaptive immune response and have the ability to differentiate into different $\mathrm{T}$ helper subtypes including atherosclerotic $\mathrm{T}$ cells (TH1 and TH17 cells) and protective cells (TH2 and regulatory T [Treg] cells) Among these, TH17 secretes signature cytokine IL$17 \mathrm{~A}$ and has a role in triggering immune inflammation and promoting AS. Natural Treg cells (nTreg cells) express factor forkhead box protein P3 (Foxp3) and can negatively control immune responses (Foks et al., 2011; Tse et al., 2013; Song and
Yang, 2017; Gisterå and Hansson, 2017). Ample literature has documented there is a Th17/Treg imbalance in patients with coronary artery AS, accompaned by a significant increase in Th17 and a decrease in Treg cells (Li et al., 2014; Potekhina et al., 2015). There is an increasing effort to identify immunemodulating therapies targeting immune cells with a potential anti-atherosclerotic impact. Convincing evidence indicates that baicalin could exert its anti-arteriosclerosis effects mainly by balancing Th17/Treg cells. As mentioned before, Foxp3 could release anti-inflammatory cytokines such as IL-10 and TGF- $\beta 1$, which plays a crucial role in the differentiation of Treg cells (Marson et al., 2007; Ait-Oufella et al., 2009). There is an investigation indicating that baicalin could markedly induce Foxp3 expression and increase Treg cells, as well as increase the levels of two relational serum cytokines (TGF- $\beta 1$ and IL-10) in animal models (Liao et al., 2014). Similarly, Yang et al. found that baicalin up-regulated both exogenous and endogenous Foxp3 expression and promoted Treg cell differentiation in vitro using HEK 293 T cells as a cell model (Yang et al., 2012). In their previous studies, the same authors also confirmed that baicalin could inhibit the differentiation of Th17 cells in vivo and in vitro. The mechanism might be closely associated with baicalin inhibition the IL-6 and RORct $\gamma$ expression (Yang et al., 2011). Baicalin works by regulating the immune balance of Th17/Treg cells. The above evidences support the concept that baicalin can improve AS through immunomodulation, which may represent an important mechanism underlying the anti-atherosclerosis effects of baicalin. Apart from influencing adaptive immune cells, baicalin had been reported to also inhibit the production of red blood cell immunity, especially IgG production, by regulating the Treg/Th17 axis, but the correlation between red blood cell immunity with AS is unclear (Jiang et al., 2019). In short, the therapeutic utility of baicalin in AS at least partly ascribed to the regulation of immunomodulatory effects.

\section{Suppression of VSMC Proliferation and Migration}

Abnormal proliferation and migration of vascular smooth muscle cells (VSMCs) lead to intimal focal fibrous thickening and atherosclerotic plaque formation, while it is worth noting that VSMCs in advanced plaques are entirely beneficial. For example, VSMCs preventing rupture of the fibrous cap. Smooth muscle (SM)22, a differentiated VSMC marker, is a cytoskeletonassociated protein and is important for maintaining the differentiated phenotype of VSMCs (Fu et al., 2000; Han et al., 2009; Dong et al., 2012). Disruption of SM22 $\alpha$ is known to increase atherosclerotic lesions and enhance arterial proinflammation (Shen et al., 2010; Shu et al., 2015). Baicalin has been reported to up-regulate the SM22 $\alpha$ which may represent a safe and effective approach to prevent vascular disease (Lv et al., 2016). Moreover, baicalin is able to inhibit the proliferation of VSMCs by repressing cell cycle progression and arresting the human aorta VSMCs cycle at the G0/G1 phase. The molecular mechanism of this effect was associated with activating the expression of maternally expressed gene 3 (MEG3), which is a long-chain non-coding RNA, whose transcriptional deficiency increases cell cycle-associated protein 
expression (Liu et al., 2019). Furthermore, baicalin could activate the p53 signaling pathway and promoted the expression and transport of p53 leading to apoptosis of VSMCs. Overall, based on an in vitro model of AS, baicalin inhibited proliferation and promoted apoptosis in ox-LDL-treated HA-VSMCs by activating the expression of MEG3/p53 (Liu et al., 2019). Furthermore, baicalin has also been reported to suppress the proliferation and migration of ox-LDL-VSMCs by upregulating a family of endogenous, small and non-coding RNAs called miR-126-5p by targeting high-mobility group box 1 (HMGB1) (Chen et al., 2019). Thus, it is apparent that blockade of VSMC proliferation and migration are important constituents of the atheroprotective effects of baicalin.

\section{Myocardial Ischemia-Reperfusion Injury (MIRI)}

Ischemic heart disease is primarily caused by coronary AS and its complications. MI is the most common cause of ischemic heart disease (Pasupathy et al., 2015). Reperfusion is mandatory to salvage the ischemic myocardium from infarction, but reperfusion per se contributes to injury and ultimate infarct size (Reimer et al., 1993; Vandervelde et al., 2006; FernándezJiménez et al., 2015). Therefore, cardioprotection beyond that by timely reperfusion is needed to reduce infarct size and to improve the prognosis of patients with acute myocardial infarction. Numerous studies have confirmed that baicalin has a protective effect on the infarcted myocardium involved in myocardial infarction and myocardial ischemia-reperfusion, and its main mechanism of action includes inflammation regulation, inhibition of oxidative stress and reduction of apoptosis (Lin et al., 2010; Kong et al., 2014).

\section{Mitigation of Inflammatory Response}

MI initiates an intense inflammatory response, while inflammation is an important process in the pathophysiological myocardial $I / R$ injury (Frangogiannis, 2014; Ma et al., 2014; Yang et al., 2016). Under inflammatory conditions, signaling pathways like mitogenactivated protein kinases (MAPKs) and NF- $\kappa \mathrm{B}$ are activated, aggravating large amounts of pro-inflammatory markers such as TNF- $\alpha$, IL- $1 \beta$, IL-6, and IL-18, and anti-inflammatory cytokines such as IL-10 which protect cardiac function (Chandrasekar et al., 2000; Hall et al., 2006). MAPK cascades, especially extracellular signal-regulated kinase (ERK), c-Jun N-terminal kinase/stressactivated protein kinase (JNK/SAPK) and p38, are expresses in the myocardium and play a pivotal role in the amelioration of ischemic insults (Yue et al., 2000; Petrich and Wang, 2004). Current work has shown that the phosphorylated (p)-ERK was reduced while the p-JNK and p-p38 were elevated in the rat model of acute MI (Liu et al., 2013). Therefore, targeting the inflammatory cascade and related inflammatory cytokines is crucial in improving myocardial ischemia and reperfusion.

Baicalin plays a protective role in ischemia/reperfusion (I/R) myocardial injury and protects against hypoxia/reoxygenation $(\mathrm{H} / \mathrm{R})$ damage. A previous report confirmed that baicalin protected against I/R injury in cultured chick cardiomyocytes (Chang et al., 2006). In terms of the baicalin on the anti- inflammatory mechanisms, on one hand, baicalin's cardioprotection was associated with mediation of MAPKs cascades. In the acute MI (AMI) model of rats, which following treatment with baicalin treatment, Liu and colleagues found that the cardioprotective effect of baicalin might be achieved via the activation of ERK and suppression of JNK and p38 activity. They also showed that baicalin played a favorable role against AMI impairment by decreasing myocardial injury marker such as the creatine kinase $(\mathrm{CK})$, creatine kinase $\mathrm{MB}(\mathrm{CK}-\mathrm{MB})$, lactate dehydrogenase (LDH) and serum cardial troponin $\mathrm{T}(\mathrm{cTnT})$ as well as reducing the infarction size (Liu et al., 2013). Similarly, results from the MI rats model induced by isoproterenol, not only did baicalin ameliorated infarct size and CK, CK-MB, LDH and cTnT levels, but it also suppressed p-38 protein expressions significantly (Sun et al., 2015). Conversely, baicalin treatment effectively inhibited the NF- $\mathrm{B}$ pathway and exerted cardioprotective effects in the cultured rat cardiomyocytes exposed to H/R (Lin et al., 2010). The phosphatidylinositol 3kinase (PI3K)/Akt signaling pathways also participates in the regulation of $\mathrm{NF}-\mathrm{\kappa B}$ in the inflammatory response. In the male rats $\mathrm{I} / \mathrm{R}$ model, intragastric administration of baicalin could attenuate I/R-induced myocardial damage via activating PI3K/ Akt signaling and suppressing NF- $\mathrm{KB}$ signaling (Luan et al., 2019). In addition, among these studies, regulation of the levels of inflammatory cytokines was almost consistent. With the treatment of baicalin, pro-inflammatory markers such as TNF$\alpha$, IL-6, IL-1 $\beta$ and IL- 8 in myocardial tissues were downregulated, and the anti-inflammatory cytokine, IL-10, was upregulated. Thus, according to the above findings, it is apparent that baicalin may directly suppress the inflammatory response and may then improve inflammatory-elicited myocardial injury.

\section{Inhibition of Oxidative Stress}

At least half of myocardial damage resulting from MI is associated with myocardial reperfusion injury. Once the blood supply to an organ is interrupted (ischemia) and re-established (reperfusion), this kind of situation leads to a "burst" of ROS generation from mitochondria including uncoupled SOD and MDA (Cadenas et al., 2010; Hausenloy and Yellon, 2013). There is evidence indicating that baicalin can improve ventricular function on $\mathrm{I} / \mathrm{R}$ injury in isolated rat hearts, the mechanisms of which may be associated with increasing SOD and decreasing MDA activity (Kong et al., 2014). Furthermore, in a rat model of $\mathrm{MI}$, a decrease in MDA and an increase in of SOD were observed in the baicalin treatment group (Wang L. et al., 2018). Therefore, baicalin supplementation should be considered as an effective approach for attenuating reperfusion-exerted microvascular damage. In addition, Nrf2, a redox-sensitive transcription factor, is crucial to inhibit oxidative stress in cells (Gallorini et al., 2015). Once activated, Nrf2 can transactivate genes driven by antioxidant response elements (ARE), especially HO-1 (Tong and Zhou, 2017). Herein, it has been reported that the Nrf2/HO-1 pathway exhibits protective roles in ischemic disorders, including MI (Zeng et al., 2015). One in vitro study demonstrated that baicalin treatment activated the $\mathrm{Nrf} 2 / \mathrm{HO}-1$ 
pathway in $\mathrm{H} 9 \mathrm{c} 2$ cells under hypoxic condition via further enhancing Nrf2 and HO-1 expression (Yu et al., 2019). Taken together, baicalin may be a potential preventive and therapeutic compound for improving MI.

\section{Anti-Apoptosis Activity}

Apoptosis, is involved in MI evolution, prognosis and outcome, constitutes an important form of cardiac cell death after MI (Akasaka et al., 2006; Chen et al., 2014). Inhibition of myocardial cell apoptosis can reduce the I/R injury, which is one of the clinical significance for the treatment of MI (Luan et al., 2019). Baicalin pretreatment protects against myocardial I/R injury by inhibiting mitochondrial damage-mediated apoptosis. Baicalin also exerts cardioprotective effects via regulating several signaling pathways and apoptosis regulators. The $\mathrm{Wnt} / \beta$ catenin signaling pathway is associated with ischemic heart disease. Knockdown of $\beta$-catenin expression inhibited $\mathrm{H}_{2} \mathrm{O} 2$ induced cardiomyocyte apoptosis. Baicalin could significantly downregulate the expression of $\beta$-catenin in $\mathrm{H} 2 \mathrm{O} 2$-treated $\mathrm{H} 9 \mathrm{c} 2$ cells. It was speculated that baicalin might inhibit the $\mathrm{Wnt} / \beta$ catenin signaling pathway and thus inhibit cell apoptosis, allowing it to exert a cardioprotective role. However, whether it is directly related to $\mathrm{MI}$ and reperfusion remains to be verified (Zhang et al., 2009; Gessert and Kühl, 2010; Dohn and Waxman, 2012; Qiu et al., 2017). The PI3K/Akt may also be regulated by baicalin to inhibit I/R-induced cardiomyocyte apoptosis thereby reduction of myocardial damage. Caspase- 3 is an important apoptosis executors in the caspase family, and up-regulating the expression of caspase-3 gene promotes AMI myocardial apoptosis (Zidar et al., 2007; Prech et al., 2010; Chen et al., 2014). Cell-based research indicated that baicalin could inhibit caspase-3 activity and protein expression to effectively protect the heart from MI damage. The specific mechanism involved might be related to the activation of survival kinases including ERK and the inhibition of apoptotic kinases such as JNK and p38 (Sun et al., 2015). Bax and Bcl-2 belong to the same apoptosis gene family, but they have opposite effects. Bcl-2 inhibits cell apoptosis, while Bax promotes it (Karsan et al., 1997). Baicalin could improve MI by up-regulating the expression of Bcl-2 and down-regulating Bax (Wang L. et al., 2018). Similarly, the results of another in vitro experiments also implied that pretreatment with baicalin significantly reduced cytochrome c, Bax and increased Bcl-2 expression in H/R-induced cardiomyocytes (Jiang et al., 2018).

Furthermore, mitochondrial injury-mediated apoptosis is also an important baicalin mechanism potentially able to protect against myocardial $\mathrm{I} / \mathrm{R}$ injury. It is well known that cardiomyocyte apoptosis, resulting from mitochondrial dysfunction, is considered to be a major contributor to $\mathrm{I} / \mathrm{R}$ injury (Wang et al., 2013). The most representative is mitochondrial aldehyde dehydrogenase 2 (ALDH2), which is widely expressed in the heart, is an anti-apoptotic enzyme that participates in oxidative stress-induced cell apoptosis (Zhang et al., 2011; Sun et al., 2014). ALDH2 maintains mitochondrial function and inhibits ROS generation (Vander Heide and Steenbergen, 2013). Emerging evidence has revealed that ALDH2 has a cardioprotective role in myocardial IR injury
(Pang et al., 2015). The study by Jiang et al. demonstrated that baicalin could reduce the apoptosis and oxidative stress by enhancing the expression and activity of ALDH2 to protect against the cardioprotective effect of H/R-induced H9c2 cell damage (Jiang et al., 2018). Taken together, understanding the effects of baicalin in mediating functions of anti-apoptotic activity would supply newly insights into the understanding for the treatment of MIRI.

\section{Hypertension}

Hypertension is a complex disease that involves an interaction between environmental factors, genetic influences and abnormalities in regulatory mechanisms of the cardiovascular system (Parati, 2015; Hoffmann et al., 2017; Calvillo et al., 2019). Cumulative findings support the notion that inflammation, oxidative stress and endothelial dysfunction leads to the development of hypertension (Dinh et al., 2014). Targeting these pathogenesis processes is indicated to be a critical mechanisms underlying the baicalin hypotensive effect and subsequent cardiovascular events (Ding et al., 2019).

Recently, the immune system and inflammatory response have been shown to play an essential role in the pathogenesis of hypertension. Many inflammatory markers such as C-reactive protein (CRP), cytokines, and adhesion molecules have been found to be elevated in hypertensive patients supporting the role of inflammation in the pathogenesis of hypertension. CRP could stimulate monocytes to release proinflammatory cytokines such as IL-6, IL-1 $\beta$, and TNF- $\alpha$ which will further promote inflammation (Crowley, 2014). In vivo, experimental data confirmed that baicalin forcefully lowered the blood pressure partly via decreasing the serum levels of high-sensitivity CRP, IL-6 and IL-1 $\beta$ in spontaneously hypertensive rats (SHRs) (Wu et al., 2019). Similarly, another vitro study showed that baicalin extenuated contents of TNF- $\alpha$ by altering miR-145 expression and significantly ameliorating TNF- $\alpha$ inflammatory injuries in human aortic endothelial cells (HAECs) (Li et al., 2020). With the popularization of the Holistic concept, numerous studies have revealed that a damage in the intestinal barrier, is considered as a critical role in the pathogenesis of hypertension primarily by exacerbating the development of chronic low-grade inflammation (Santisteban et al., 2017; Jaworska et al., 2017). The increment of intestinal permeability caused by intestinal barrier dysfunction allows leakage of bacterial metabolites into the circulation. One such metabolite is bacterial lipopolysaccharide, a TLR4 ligand, which is increased in the circulation of patients with hypertension (Kim et al., 2018). One of an important consequence of TLR signaling is the priming of inflammasomes. Various inflammasome-derived cytokine IL- $1 \beta$ or/and IL- 6 is augmented in several blood pressure-regulating sites during hypertension, including medial and adventitial layers of arteries (Krishnan et al., 2016; Zhu Q. et al., 2016). Existing study proves that baicalin take the protective effects on the intestinal integrity in the SHRs. One molecular evidence is that baicalin could decreases the serum levels of inflammatory indicators such as high-sensitivity Creactive protein (hs-CRP), IL-1 1 , and IL- 6 . Another one is that baicalin increases the expression of tight junction proteins such 


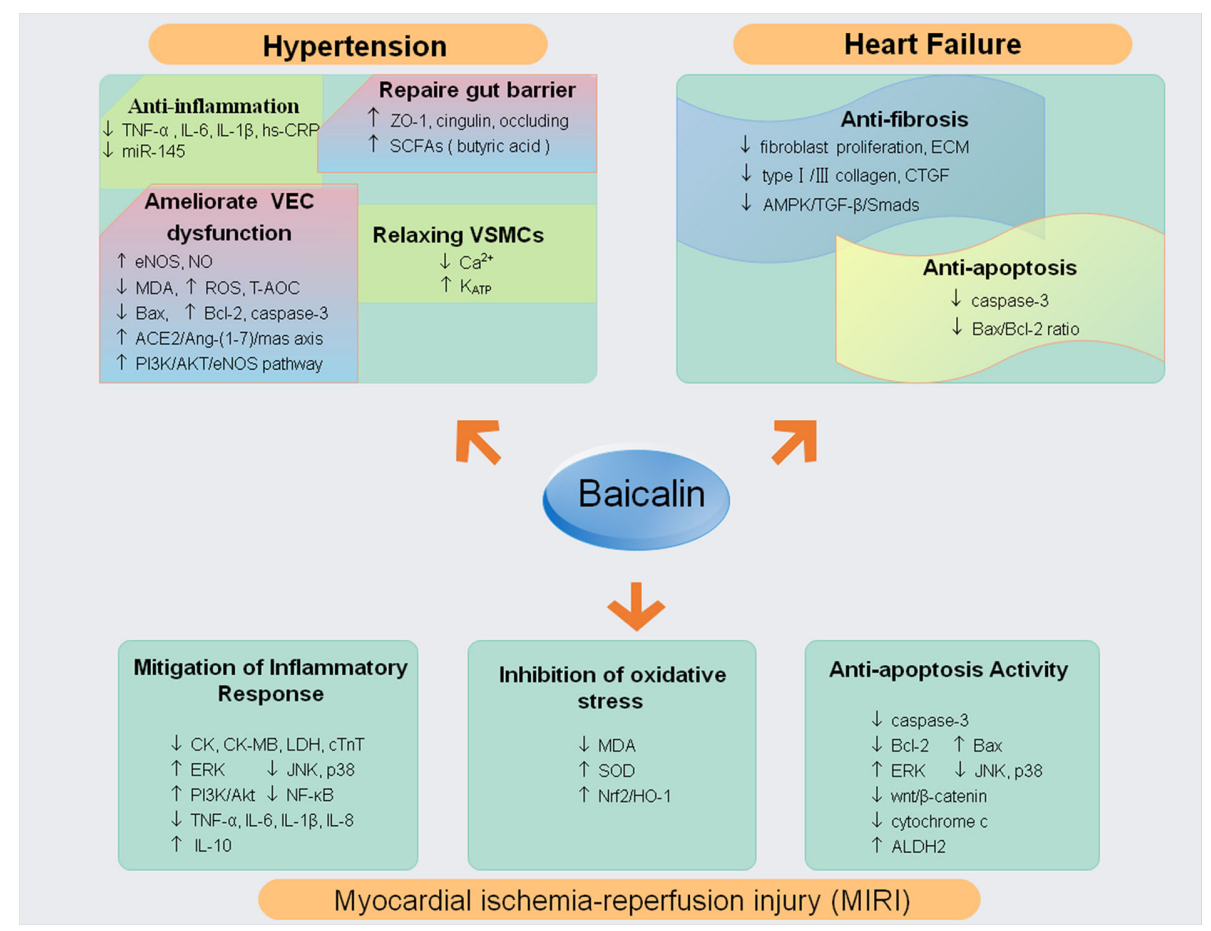

FIGURE 4 | The molecular mechanisms and potential targets of baicalin in hypertension, myocardial infarction and reperfusion, heart failure ( $\downarrow$ decrease or inhibit; $\uparrow$ increase or up-regulate). Abbreviations: tumor necrosis factor (TNF)- $\alpha$; extracellular signal-regulated kinase (ERK); c-Jun N-terminal kinase (JNK); phosphatidylinositol 3-kinase (PI3K); nuclear factor kappa B (NF-kB); interleukin 6, 8, 10, 1 ( (IL-6, IL-1ß, IL-8,IL-10); superoxide dismutase (SOD); malondialdehyde (MDA); nuclear factor E2-associated factor 2 (Nrf2); heme oxygenase-1 (HO-1); mitogen-activated protein kinases (MAPKs); mitochondrial aldehyde dehydrogenase 2 (ALDH2); highsensitivity C-reactive protein (hs-CRP); zonula occludens-1 (ZO-1); Short-chain fatty acids (SCFAs); oxygen reactive species (ROS);(eNOS);Total Antioxidant Capacity (T-AOC); nitric oxide (NO); extracellular matrix (ECM); Connective Tissue Growth Factor (CTGF); creatine kinase (CK), creatine kinase MB (CK-MB), lactate dehydrogenase (LDH) and serum cardial troponinT (CTnT); malondialdehyde (MDA); superoxide dismutase (SOD).

as zonules occludin-1 (ZO-1), cingulin and occluding. Besides, short-chain fatty acids (SCFAs), as the metabolites of intestinal microbes, also play an important role in the integrity of the intestines, and their pathway of action may be related to enhancing the expression tight junction proteins ( $\mathrm{Wu}$ et al., 2019). A pivotal study shows that baicalin treatment blunts the development of experimental hypertension partly by promoting the production of fecal SCFAs and the abundance of SCFAproducing bacterial in the SHRs. Thus, all these studies support the protective effects of baicalin on the intestinal integrity in the treatment of hypertension. According to previous studies, sustained inflammation contributes to the overproduction of ROS which in turn aggravates the inflammatory process and perturbs the function of the vasculature (Crowley, 2014). In the pathogenesis of hypertension, Angiotensin II (Ang II), as the main active peptide of renin-angiotensin system (RAS), can participate in oxidative stress and apoptosis leading to endothelial dysfunction by activating apoptosis-related proteins, stimulating ROS production, and reducing NO production. Therefore, interfering with Ang II-induced endothelial dysfunction, inhibiting oxidative stress, and reducing cell apoptosis can effectively improve hypertension. A study involving the HUVEC model of Ang II injury determined that baicalin can significantly reduce the endothelial dysfunction and oxidative stress induced by Ang II. These positive effects are mainly achieved by regulating the expression of Bax, Bcl-2 and cleaved caspase-3, activating the ACE2/Ang-(1-7)/mas axis, and upregulating the PI3K/AKT/eNOS pathway. They also found that baicalin attenuated oxidative stress indicators such as reducing MDA and ROS, promoting nitric oxide (NO) and Total Antioxidant Capacity (T-AOC) levels (Heitsch et al., 2001; Mehta and Griendling, 2007; Sampaio et al., 2007; Wei et al., 2015). Another potential mechanism involved in the hypertensionprotecting effects of baicalin is enhancing endothelial nitric oxide synthase (eNOS) -induced production of endogenous NO in HUVECs (Chen et al., 2013). Apart from the above pathogenetic mechanisms involving baicalin, baicalin lowered blood pressure partially by relaxing vascular smooth muscle by decreasing $\mathrm{Ca}^{2+}$ levels and the enhancing $\mathrm{K}_{\mathrm{ATP}}$ function in VSMCs (Ding et al., 2019). The combined application of baicalin and berberine was also found to relax blood vessels, owing to the voltage-dependent $\mathrm{Ca}^{2+}$ channel (VDCC) (Wu et al., 2020). Together, these studies indicated that baicalin might have comprehensive effects on ameliorating hypertension. 


\section{Heart Failure}

Heart failure (HF) is the final process resulting from different cardiac insults and subsequent dysregulation of in compensatory mechanisms and pathogenic processes (Yancy et al., 2013). Cardiac fibrosis characterized by interstitial fibroblast proliferation and excessive production and deposition of myocardial extracellular matrix (ECM) proteins is an independent and predictive risk factor for $\mathrm{HF}$ in both ischemic and nonischemic cardiomyopathy (Fan et al., 2012; Prabhu and Frangogiannis, 2016). Some studies have revealed that baicalin had an anti-fibrosis effect. One study had observed that baicalin could alleviate myocardial fibrosis manifested by reducing the ECM and decreasing fibrosis genes expression [type I collagen, type III collagen and Connective Tissue Growth Factor (CTGF)] in pressure overload mouse model (Zhang et al., 2017). Similarly, another study found that baicalin could inhibit fibroblast proliferation and ECM accumulation, thereby suppressing cardiac fibrosis from the pressure overload-induced in the abdominal aortic constriction (AAC) rat model; the underlying mechanisms are linked to the AMPK/TGF- $\beta /$ Smads signaling pathway (Xiao et al., 2018). Moreover, baicalin inhibited apoptosis by reducing the $\mathrm{Bax} / \mathrm{Bcl}-2$ ratio and caspase-3, indicating that that suppression of apoptosis could decrease adverse remodeling and subsequent progression to $\mathrm{HF}$ (Wencker et al., 2003; Dai et al., 2017). It is worth exploring additional more mechanisms of baicalin-mediated HF protection furtherly (Figure 4).

\section{CONCLUSION}

In conclusion, numerous preclinical studies have provided evidence that baicalin, a naturally occurring bioactive compound in S. baicalensis Georgi, is a promising therapeutic agent for cardiovascular protection. The pharmacokinetics profile of baicalin mainly includes gastrointestinal hydrolysis, enterohepatic recycling, carrier-mediated transport and complicated metabolism. A comprehensive understanding of its pharmacokinetics is essential for its safety and efficacy in clinical applications. Baicalin exerts prophylactic and/or therapeutic effects in cardiovascular disorders via mechanisms involving in regulating lipid metabolism, reducing inflammation-induced damage, inhibiting oxidative stress,

\section{REFERENCES}

Abe, K., Inoue, O., and Yumioka, E. (1990). Biliary excretion of metabolites of baicalin and baicalein in rats. Chem. Pharm. Bull. 38 (1), 209-211. doi: 10.1248/ cpb.38.208

Aggarwal, M., Aggarwal, B., and Rao, J. (2017). Integrative Medicine for Cardiovascular Disease and Prevention. Med. Clinics North America 101 (5), 895-923. doi: 10.1016/j.mcna.2017.04.007

Ait-Oufella, H., Taleb, S., Mallat, Z., and Tedgui, A. (2009). Cytokine network and T cell immunity in atherosclerosis. Semin. Immunopathol. 31 (1), 23-33. doi: 10.1007/s00281-009-0143-x

Akao, T., Sato, K., He, J. X., Ma, C. M., and Hattori, M. (2013). Baicalein 6-O- $\beta$-Dglucopyranuronoside is a main metabolite in the plasma after oral administration of baicalin, a flavone glucuronide of scutellariae radix, to rats. Biol. Pharm. Bull. 36 (5), 748-753. doi: 10.1248/bpb.b12-00850 reducing apoptosis, and immune regulation. The pleiotropic pharmacological activities of baicalin suggest it has great potential for clinical application in the prevention and treatment of AS, MIRI, hypertension, and HF. Although a great deal of knowledge has been acquired regarding the benefits of baicalin on CVDs from experimental data, it is worth noting that the specific underlying mechanisms are still relatively unexplained. Thus, the limited clinical and pharmacological data available are not enough to evaluate its efficacy at the present moment. Further, concerning the undesirable physical characteristics of baicalin, additional research and technological development are required to improve bioavailability and to overcome the challenges in its clinical application. Therefore, making full use of modern analytical techniques, establishing reasonable detection methods, and studying the role of baicalin in a more systematic and in-depth manner are important. Further exploration into the molecular mechanisms and potential targets of baicalin, is also urgent and necessary in order to conduct a large randomized and controlled trials to evaluate the efficacy and safety of the cardiovascular activity of baicalin.

\section{AUTHOR CONTRIBUTIONS}

LY-X wrote the main text. JL-G contributed equally to this work. HC-L, YQ, CS, and YL-W retrieved and organized documents. $\mathrm{YD}-\mathrm{L}$ and $\mathrm{XN}-\mathrm{C}$ had great contribution in second time revision, polishing manuscript, and helping in revising figures.

\section{FUNDING}

This work was funded by grants from the National Natural Science Foundation of China (No.81973842).

\section{ACKNOWLEDGMENTS}

We thank Y. D. Lu, J. L. Gao, and X. N. Cui for the guidance and revision of this manuscript.

Akasaka, Y., Morimoto, N., Ishikawa, Y., Fujita, K., Ito, K., KimuraMatsumoto,, et al. (2006). Myocardial apoptosis associated with the expression of proinflammatory cytokines during the course of myocardial infarction. Modern Pathol. 19 (4), 588-598. doi: 10.1038/modpathol. 3800568

Ammirati, E., Moroni, F., Magnoni, M., and Camici, P. G. (2015). The role of T and $\mathrm{B}$ cells in human atherosclerosis and atherothrombosis. Clin. Exp. Immunol. 179 (2), 173-187. doi: 10.1111/cei.12477

Azimova, S. S., and Vinogradova, V. I. (2013). "Physicochemical and pharmacological properties of flavonoids," in Natural compounds-flavonoids (New York (NY: Springer), p.86-p.87.

Bäck, M., Yurdagul, A. C.OMMAJ.R.X.X.X, Tabas, I., Öörni, K., and Kovanen, P. T. (2019). Inflammation and its resolution in atherosclerosis: mediators and therapeutic opportunities. Nat. Rev. Cardiol. 16 (7), 389-406. doi: 10.1038/ s41569-019-0169-2 
Bonnes-Taourel, D., Guérin, M. C., and Torreilles, J. (1992). Is malonaldehyde a valuable indicator of lipid peroxidation? Biochem. Pharmacol. 44 (5), 985-988. doi: 10.1016/0006-2952(92)90132-3

Cadenas, S., Aragonés, J., and Landázuri, M. O. (2010). Mitochondrial reprogramming through cardiac oxygen sensors in ischaemic heart disease. Cardiovasc. Res. 88 (2), 219-228. doi: 10.1093/cvr/cvq256

Calvillo, L., Gironacci, M. M., Crotti, L., Meroni, P. L., and Parati, G. (2019). Neuroimmune crosstalk in the pathophysiology of hypertension. Nat. Rev. Cardiol. 16 (8), 476-490. doi: 10.1038/s41569-019-0178-1

Chandrasekar, B., Colston, J. T., Geimer, J., Cortez, D., and Freeman, G. L. (2000). Induction of nuclear factor kappaB but not kappaB-responsive cytokine expression during myocardial reperfusion injury after neutropenia. Free Radical Biol. Med. 28 (11), 1579-1588. doi: 10.1016/s0891-5849(00)00273-2

Chang, W. T., Shao, Z. H., Vanden Hoek, T. L., McEntee, E., Mehendale, S. R., Li, J., et al. (2006). Cardioprotective effects of grape seed proanthocyanidins, baicalin and wogonin: comparison between acute and chronic treatments. Am. J. Chin. Med. 34 (2), 363-365. doi: 10.1142/S0192415X06003898

Chawla, A., Boisvert, W. A., Lee, C. H., Laffitte, B. A., Barak, Y., Joseph, S. B., et al. (2001). A PPAR gamma-LXR-ABCA1 pathway in macrophages is involved in cholesterol efflux and atherogenesis. Mol. Cell 7 (1), 161-171. doi: 10.1016/ s1097-2765(01)00164-2

S. L. Chen, M. G. Gilbert and X. W. Li (Eds.) (1994). Flora of China Vol. 17 (St. Louis, MO, U.S.A.: Missouri Botanical Garden Press), 93.

Chen, Z., Nihei, K., Tanaka, H., Uda, Y., and Kabuyama, Y. (2013). Identification of a nitric oxide generation-stimulative principle in Scutellariae radix. Biosci. Biotechnol. Biochem. 77 (3), 657-659. doi: 10.1271/bbb.120800

Chen, T. L., Zhu, G. L., He, X. L., Wang, J. A., Wang, Y., and Qi, G. A. (2014). Short-term pretreatment with atorvastatin attenuates left ventricular dysfunction, reduces infarct size and apoptosis in acute myocardial infarction rats. Int. J. Clin. Exp. Med. 7 (12), 4799-4808.

Chen, H., Xu, Y., Wang, J., Zhao, W., and Ruan, H. (2015). Baicalin ameliorates isoproterenol-induced acute myocardial infarction through iNOS, inflammation and oxidative stress in rat. Int. J. Clin. Exp. Pathol. 8 (9), 10139-10147.

Chen, Y., Minh, L. V., Liu, J., Angelov, B., Drechsler, M., Garamus, V. M., et al. (2016). Baicalin loaded in folate-PEG modified liposomes for enhanced stability and tumor targeting. Colloids Surf. B. Biointerf. 140, 74-82. doi: 10.1016/j.colsurfb.2015.11.018

Chen, Z., Pan, X., Sheng, Z., Yan, G., Chen, L., and Ma, G. (2019). Baicalin Suppresses the Proliferation and Migration of Ox-LDL-VSMCs in Atherosclerosis through Upregulating miR-126-5p. Biol. Pharm. Bull. 42 (9), 1517-1523. doi: 10.1248/bpb.b19-00196

Chistiakov, D. A., Melnichenko, A. A., Grechko, A. V., Myasoedova, V. A., and Orekhov, A. N. (2018). Potential of anti-inflammatory agents for treatment of atherosclerosis. Exp. Mol. Pathol. 104 (2), 114-124. doi: 10.1016/ j.yexmp.2018.01.008

Collot-Teixeira, S., Martin, J., McDermott-Roe, C., Poston, R., and McGregor, J. L. (2007). CD36 and macrophages in atherosclerosis. Cardiovasc. Res. 75 (3), 468-477. doi: 10.1016/j.cardiores.2007.03.010

Crowley, S. D. (2014). The cooperative roles of inflammation and oxidative stress in the pathogenesis of hypertension. Antioxid. Redox Signaling 20 (1), 102-120. doi: 10.1089/ars.2013.5258

Dai, H., Zhang, X., Yang, Z., Li, J., and Zheng, J. (2017). Effects of Baicalin on Blood Pressure and Left Ventricular Remodeling in Rats with Renovascular Hypertension. Med. Sci. Monitor Int. Med. J. Exp. Clin. Res. 23, 2939-2948. doi: $10.12659 / \mathrm{msm} .902536$

Dinda, B., Dinda, S., DasSharma, S., Banik, R., Chakraborty, A., and Dinda, M. (2017). Therapeutic potentials of baicalin and its aglycone, baicalein against inflammatory disorders. Eur. J. Med. Chem. 131, 68-80. doi: 10.1016/ j.ejmech.2017.03.004

Ding, L., Jia, C., Zhang, Y., Wang, W., Zhu, W., Chen, Y., et al. (2019). Baicalin relaxes vascular smooth muscle and lowers blood pressure in spontaneously hypertensive rats. Biomed. Pharmacother. Biomed. Pharmacother. 111, 325330. doi: 10.1016/j.biopha.2018.12.086

Dinh, Q. N., Drummond, G. R., Sobey, C. G., and Chrissobolis, S. (2014). Roles of inflammation, oxidative stress, and vascular dysfunction in hypertension. BioMed. Res. Int. 2014, 406960. doi: 10.1155/2014/406960
Dohn, T. E., and Waxman, J. S. (2012). Distinct phases of Wnt/ $\beta$-catenin signaling direct cardiomyocyte formation in zebrafish. Dev. Biol. 361 (2), 364-376. doi: 10.1016/j.ydbio.2011.10.032

Dong, L. H., Lv, P., and Han, M. (2012). Roles of SM22 $\alpha$ in cellular plasticity and vascular diseases. Cardiovasc. Hematol. Disord. Drug Targets 12 (2), 119-125. doi: 10.2174/1871529x11202020119

Fan, D., Takawale, A., Lee, J., and Kassiri, Z. (2012). Cardiac fibroblasts, fibrosis and extracellular matrix remodeling in heart disease. Fibrogen. Tissue Repair 5 (1), 15. doi: 10.1186/1755-1536-5-15

Fernández-Jiménez, R., García-Prieto, J., Sánchez-González, J., Agüero, J., LópezMartín, G. J., Galán-Arriola, C., et al. (2015). Pathophysiology Underlying the Bimodal Edema Phenomenon After Myocardial Ischemia/Reperfusion. J. Am. Coll. Cardiol. 66 (7), 816-828. doi: 10.1016/j.jacc.2015.06.023

Foks, A. C., Frodermann, V., ter Borg, M., Habets, K. L., Bot, I., Zhao, Y., et al. (2011). Differential effects of regulatory $\mathrm{T}$ cells on the initiation and regression of atherosclerosis. Atherosclerosis 218 (1), 53-60. doi: 10.1016/ j.atherosclerosis.2011.04.029

Frangogiannis, N. G. (2014). The inflammatory response in myocardial injury, repair, and remodelling. Nat. Rev. Cardiol. 11 (5), 255-265. doi: 10.1038/nrcardio.2014.28

Fu, Y., Liu, H. W., Forsythe, S. M., Kogut, P., McConville, J. F., Halayko, A. J., et al. (2000). Mutagenesis analysis of human SM22: characterization of actin binding. J. Appl. Physiol. (Bethesda Md. 1985) 89 (5), 1985-1990. doi: 10.1152/jappl.2000.89.5.1985

Gallorini, M., Petzel, C., Bolay, C., Hiller, K. A., Cataldi, A., Buchalla, W., et al. (2015). Activation of the Nrf2-regulated antioxidant cell response inhibits HEMA-induced oxidative stress and supports cell viability. Biomaterials 56, 114-128. doi: 10.1016/j.biomaterials.2015.03.047

Gargiulo, S., Gramanzini, M., and Mancini, M. (2016). Molecular Imaging of Vulnerable Atherosclerotic Plaques in Animal Models. Int. J. Mol. Sci. 17 (9), 1511. doi: 10.3390/ijms17091511

Gessert, S., and Kühl, M. (2010). The multiple phases and faces of wnt signaling during cardiac differentiation and development. Circ. Res. 107 (2), 186-199. doi: 10.1161/CIRCRESAHA.110.221531

Gherghe, C. M., Duan, J., Gong, J., Rojas, M., Klauber-Demore, N., Majesky, M., et al. (2011). Wnt1 is a proangiogenic molecule, enhances human endothelial progenitor function, and increases blood flow to ischemic limbs in a HGFdependent manner. FASEB J. Off. Publ. Fed. Am. Soc. Exp. Biol. 25 (6), 18361843. doi: 10.1096/fj.10-172981

Gisterå, A., and Hansson, G. K. (2017). The immunology of atherosclerosis. Nat. Rev. Nephrol. 13 (6), 368-380. doi: 10.1038/nrneph.2017.51

Gu, X., Kozarsky, K., and Krieger, M. (2000). Scavenger receptor class B, type Imediated $[3 \mathrm{H}]$ cholesterol efflux to high and low density lipoproteins is dependent on lipoprotein binding to the receptor. J. Biol. Chem. 275 (39), 29993-30001. doi: 10.1074/jbc.275.39.29993

Guo, X. Y., Liu, J., Liu, J., Li, H. J., Qi, Y., Qin, L. P., et al. (2013). Use of traditional Chinese medicine in Chinese patients with coronary heart disease. Biomed. Environ. Sci.: BES 26 (4), 303-310. doi: 10.3967/0895-3988.2013.04.009

Hall, G., Hasday, J. D., and Rogers, T. B. (2006). Regulating the regulator: NFkappaB signaling in heart. J. Mol. Cell. Cardiol. 41 (4), 580-591. doi: 10.1016/ j.yjmcc.2006.07.006

Han, M., Dong, L. H., Zheng, B., Shi, J. H., Wen, J. K., and Cheng, Y. (2009). Smooth muscle 22 alpha maintains the differentiated phenotype of vascular smooth muscle cells by inducing filamentous actin bundling. Life Sci. 84 (1314), 394-401. doi: 10.1016/j.lfs.2008.11.017

Hang, Y., Qin, X., Ren, T., and Cao, J. (2018). Baicalin reduces blood lipids and inflammation in patients with coronary artery disease and rheumatoid arthritis: a randomized, double-blind, placebo-controlled trial. Lipids Health Dis. 17 (1), 146. doi: 10.1186/s12944-018-0797-2

Hansson, G. K., and Hermansson, A. (2011). The immune system in atherosclerosis. Nat. Immunol. 12 (3), 204-212. doi: 10.1038/ni.2001

Hassan, W., Rongyin, G., Daoud, A., Ding, L., Wang, L., Liu, J., et al. (2014). Reduced oxidative stress contributes to the lipid lowering effects of isoquercitrin in free fatty acids induced hepatocytes. Oxid. Med. Cell. Longevity 2014, 313602. doi: 10.1155/2014/313602

Hausenloy, D. J., and Yellon, D. M. (2013). Myocardial ischemia-reperfusion injury:a neglected therapeutic target. J. Clin. Invest. 123 (1), 92-100. doi: $10.1172 /$ JCI62874 
He, X. W., Yu, D., Li, W. L., Zheng, Z., Lv, C. L., Li, C., et al. (2016). Antiatherosclerotic potential of baicalin mediated by promoting cholesterol efflux from macrophages via the PPAR $\gamma$-LXR $\alpha$-ABCA1/ABCG1 pathway. Biomed. Pharmacother. Biomed. Pharmacother. 83, 257-264. doi: 10.1016/ j.biopha.2016.06.046

Heidenreich, P. A., Trogdon, J. G., Khavjou, O. A., Butler, J., Dracup, K., Ezekowitz, M. D., et al. (2011). Forecasting the future of cardiovascular disease in the United States: a policy statement from the American Heart Association. Circulation 123 (8), 933-944. doi: 10.1161/CIR.0b013e31820a55f5

Heitsch, H., Brovkovych, S., Malinski, T., and Wiemer, G. (2001). Angiotensin-(17)-Stimulated Nitric Oxide and Superoxide Release From Endothelial Cells. Hypertension (Dallas Tex.:1979) 37 (1), 72-76. doi: 10.1161/01.hyp.37.1.72

Herrero-Fernandez, B., Gomez-Bris, R., Somovilla-Crespo, B., and GonzalezGranado, J. M. (2019). Immunobiology of Atherosclerosis: A Complex Net of Interactions. Int. J. Mol. Sci. 20 (21), 5293. doi: 10.3390/ijms20215293

Hoffmann, T. J., Ehret, G. B., Nandakumar, P., Ranatunga, D., Schaefer, C., Kwok, P. Y., et al. (2017). Genome-wide association analyses using electronic health records identify new loci influencing blood pressure variation. Nat. Genet. 49 (1), 54-64. doi: 10.1038/ng.3715

Hovingh, G. K., Van Wijland, M. J., Brownlie, A., Bisoendial, R. J., Hayden, M. R., Kastelein, J. J., et al. (2003). The role of the ABCA1 transporter and cholesterol efflux in familial hypoalphalipoproteinemia. J. Lipid Res. 44 (6), 1251-1255. doi: 10.1194/jlr.M300080-JLR200

Huang, P., Gao, J. W., Shi, Z., Zou, J. L., Lu, Y. S., Yuan, Y. M., et al. (2012). A novel UPLC-MS/MS method for simultaneous quantification of rhein, emodin, berberine and baicalin in rat plasma and its application in a pharmacokinetic study. Bioanalysis 4 (10), 1205-1213. doi: 10.4155/bio.12.81

Huang, T., Liu, Y., and Zhang, C. (2019). Pharmacokinetics and Bioavailability Enhancement of Baicalin: A Review. Eur. J. Drug Metab. Pharmacokinet. 44 (2), 159-168. doi: 10.1007/s13318-018-0509-3

Huo, X. K., Wang, B., Zheng, L., Cong, H. J., Xiang, T., Wang, S. M., et al. (2017). Comparative pharmacokinetic study of baicalin and its metabolites after oral administration of baicalin and Chaiqin Qingning capsule in normal and febrile rats. J. Chromatography B. Analyt. Technol. Biomed. Life Sci. 1059, 14-20. doi: 10.1016/j.jchromb.2017.05.018

Jaworska, K., Huc, T., Samborowska, E., Dobrowolski, L., Bielinska, K., Gawlak, M., et al. (2017). Hypertension in rats is associated with an increased permeability of the colon to TMA, a gut bacteria metabolite. PloS One 12 (12), e0189310. doi: 10.1371/journal.pone.0189310

Jiang, W. B., Zhao, W., Chen, H., Wu, Y. Y., Wang, Y., Fu, G. S., et al. (2018). Baicalin protects $\mathrm{H} 9 \mathrm{c} 2$ cardiomyocytes against hypoxia/reoxygenationinduced apoptosis and oxidative stress through activation of mitochondrial aldehyde dehydrogenase 2. Clin. Exp. Pharmacol. Physiol. 45 (3), 303-311. doi: 10.1111/1440-1681.12876

Jiang, X., Liu, L., Sun, J., Yang, J., Xiang, D., Yang, Y., et al. (2019). Baicalin inhibits IgG production by regulating Treg/Th17 axis in a mouse model of red blood cell transfusion. Int. Immunopharmacol. 66, 282-287. doi: 10.1016/ j.intimp.2018.09.034

Karsan, A., Yee, E., Poirier, G. G., Zhou, P., Craig, R., and Harlan, J. M. (1997). Fibroblast growth factor-2 inhibits endothelial cell apoptosis by Bcl-2dependent and independent mechanisms. Am. J. Pathol. 151 (6), 1775-1784.

Kattoor, A. J., Pothineni, N., Palagiri, D., and Mehta, J. L. (2017). Oxidative Stress in Atherosclerosis. Curr. Atheroscl. Rep. 19 (11), 42. doi: 10.1007/s11883-0170678-6

Kim, D. H., Kim, H. K., Park, S., Kim, J. Y., Zou, Y., Cho, K. H., et al. (2006). Shortterm feeding of baicalin inhibits age-associated NF-kappaB activation. Mech. Ageing Dev. 127 (9), 719-725. doi: 10.1016/j.mad.2006.05.002

Kim, K.II, Park, K. U., Chun, E. J., Choi, S.II, Cho, Y. S., Youn, T. J., et al. (2011). A novel biomarker of coronary atherosclerosis: serum DKK1 concentration correlates with coronary artery calcification and atherosclerotic plaques. $J$. Korean Med. Sci. 26 (9), 1178-1184. doi: 10.3346/jkms.2011.26.9.1178

Kim, S., Goel, R., Kumar, A., Qi, Y., Lobaton, G., Hosaka, K., et al. (2018). Imbalance of gut microbiome and intestinal epithelial barrier dysfunction in patients with high blood pressure. Clin. Sci. (London Engl. 1979) 132 (6), 701718. doi: 10.1042/CS20180087

Kong, F., Luan, Y., Zhang, Z. H., Cheng, G. H., Qi, T. G., and Sun, C. (2014). Baicalin protects the myocardium from reperfusion-induced damage in isolated rat hearts via the antioxidant and paracrine effect. Exp. Ther. Med. 7 (1), 254-259. doi: 10.3892/etm.2013.1369

Krishnan, S. M., Dowling, J. K., Ling, Y. H., Diep, H., Chan, C. T., Ferens, D., et al. (2016). Inflammasome activity is essential for one kidney/deoxycorticosterone acetate/salt-induced hypertension in mice. Br. J. Pharmacol. 173 (4), 752-765. doi: 10.1111/bph.13230

$\mathrm{Ku}$, S. K., and Bae, J. S. (2015). Baicalin, baicalein and wogonin inhibits high glucose-induced vascular inflammation in vitro and in vivo. BMB Rep. 48 (9), 519-524. doi: 10.5483/bmbrep.2015.48.9.017

Ku, S. K., Seo, B.II, Park, J. H., Park, G. Y., Seo, Y. B., Kim, J. S., et al. (2009). Effect of Lonicerae Flos extracts on reflux esophagitis with antioxidant activity. World J. Gastroenterol. 15 (38), 4799-4805. doi: 10.3748/wjg.15.4799

Kutuk, O., and Basaga, H. (2003). Inflammation meets oxidation: NF-kappaB as a mediator of initial lesion development in atherosclerosis. Trends Mol. Med. 9 (12), 549-557. doi: 10.1016/j.molmed.2003.10.007

Lai, Y. S., Putra, R., Aui, S. P., and Chang, K. T. (2018). M2C Polarization by Baicalin Enhances Efferocytosis via Upregulation of MERTK Receptor. Am. J. Chin. Med. 46 (8), 1899-1914. doi: 10.1142/S0192415X18500957

Lee, H., Kang, R., Hahn, Y., Yang, Y., Kim, S. S., Cho, S. H., et al. (2009). Antiobesity effect of baicalin involves the modulations of proadipogenic and antiadipogenic regulators of the adipogenesis pathway. Phytother. Res. PTR 23 (11), 1615-1623. doi: 10.1002/ptr.2937

Lee, H., Bae, S., Kim, K., Kim, W., Chung, S. I., and Yoon, Y. (2010). Beta-Catenin mediates the anti-adipogenic effect of baicalin. Biochem. Biophys. Res. Commun. 398 (4), 741-746. doi: 10.1016/j.bbrc.2010.07.015

Li, B. Q., Fu, T., Gong, W. H., Dunlop, N., Kung, H., Yan, Y., et al. (2000). The flavonoid baicalin exhibits anti-inflammatory activity by binding to chemokines. Immunopharmacology 49 (3), 295-306. doi: 10.1016/s0162-3109 (00)00244-7

Li, B., Wen, M., Li, W., He, M., Yang, X., and Li, S. (2011). Preparation and characterization of baicalin-poly-vinylpyrrolidone coprecipitate. Int. J. Pharmaceut. 408 (1-2), 91-96. doi: 10.1016/j.ijpharm.2011.01.055

Li, C., Lin, G., and Zuo, Z. (2011). Pharmacological effects and pharmacokinetics properties of Radix Scutellariae and its bioactive flavones. Biopharmaceut. Drug Disposition 32 (8), 427-445. doi: 10.1002/bdd.771

Li, N., Je, Y. J., Yang, M., Jiang, X. H., and Ma, J. H. (2011). Pharmacokinetics of baicalin-phospholipid complex in rat plasma and brain tissues after intranasal and intravenous administration. Die Pharm. 66 (5), 374-377.

Li, Q., Wang, Y., Wang, Y., Chen, K., Zhou, Q., Wei, W., et al. (2014). Treg/Th17 ratio acts as a novel indicator for acute coronary syndrome. Cell Biochem. Biophysics 70 (2), 1489-1498. doi: 10.1007/s12013-014-9993-5

Li, J., Jiang, Q., Deng, P., Chen, Q., Yu, M., Shang, J., et al. (2017). The formation of a host-guest inclusion complex system between $\beta$-cyclodextrin and baicalin and its dissolution characteristics. J. Pharm. Pharmacol. 69 (6), 663-674. doi: $10.1111 /$ jphp. 12708

Li, L., Liu, M., He, L., Wang, S., and Cui, S. (2020). Baicalin relieves TNF- $\alpha$-evoked injury in human aortic endothelial cells by up-regulation of miR-145. Phytother. Res.: PTR 34 (4), 836-845. doi: 10.1002/ptr.6566

Liao, P., Liu, L., Wang, B., Li, W., Fang, X., and Guan, S. (2014). Baicalin and geniposide attenuate atherosclerosis involving lipids regulation and immunoregulation in ApoE-/- mice. Eur. J. Pharmacol. 740, 488-495. doi: 10.1016/j.ejphar.2014.06.039

Libby, P., Ridker, P. M., and Hansson, G. K. (2011). Progress and challenges in translating the biology of atherosclerosis. Nature 473 (7347), 317-325. doi: $10.1038 /$ nature 10146

Libby, P. (2002). Inflammation in atherosclerosis. Nature 420 (6917), 868-874. doi: $10.1038 /$ nature 01323

Lin, L., Wu, X. D., Davey, A. K., and Wang, J. (2010). The anti-inflammatory effect of baicalin on hypoxia/reoxygenation and TNF-alpha induced injury in cultural rat cardiomyocytes. Phytother. Res. PTR 24 (3), 429-437. doi: $10.1002 /$ ptr.3003

Liu, L. L., Gong, L. K., Wang, H., Xiao, Y., Wu, X. F., Zhang, Y. H., et al. (2008). Baicalin inhibits macrophage activation by lipopolysaccharide and protects mice from endotoxin shock. Biochem. Pharmacol. 75 (4), 914-922. doi: 10.1016/j.bcp.2007.10.009

Liu, L., Deng, Y. X., Liang, Y., Pang, X. Y., Liu, X. D., Liu, Y. W., et al. (2010). Increased oral AUC of baicalin in streptozotocin-induced diabetic rats due to 
the increased activity of intestinal beta-glucuronidase. Planta Med. 76 (1), $70-$ 75. doi: $10.1055 / \mathrm{s}-0029-1185946$

Liu, X., Gu, J., Fan, Y., Shi, H., and Jiang, M. (2013). Baicalin attenuates acute myocardial infarction of rats via mediating the mitogen-activated protein kinase pathway. Biol. Pharm. Bull. 36 (6), 988-994. doi: 10.1248/bpb.b13-00021

Liu, L., Liao, P., Wang, B., Fang, X., Li, W., and Guan, S. (2014). Oral administration of baicalin and geniposide induces regression of atherosclerosis via inhibiting dendritic cells in ApoE-knockout mice. Int. Immunopharmacol. 20 (1), 197-204. doi: 10.1016/j.intimp.2014.02.037

Liu, L., Liao, P., Wang, B., Fang, X., Li, W., and Guan, S. (2015). Baicalin inhibits the expression of monocyte chemoattractant protein- 1 and interleukin- 6 in the kidneys of apolipoprotein E-knockout mice fed a high cholesterol diet. Mol. Med. Rep. 11 (5), 3976-3980. doi: 10.3892/mmr.2015.3186

Liu, Y., Jia, L., Min, D., Xu, Y., Zhu, J., and Sun, Z. (2019). Baicalin inhibits proliferation and promotes apoptosis of vascular smooth muscle cells by regulating the MEG3/p53 pathway following treatment with ox-LDL. Int. J. Mol. Med. 43 (2), 901-913. doi: 10.3892/ijmm.2018.4009

Loboda, A., Damulewicz, M., Pyza, E., Jozkowicz, A., and Dulak, J. (2016). Role of $\mathrm{Nrf} 2 / \mathrm{HO}-1$ system in development, oxidative stress response and diseases: an evolutionarily conserved mechanism. Cell. Mol. Life Sci. CMLS 73 (17), 32213247. doi: 10.1007/s00018-016-2223-0

Lu, T., Song, J., Huang, F., Deng, Y., Xie, L., Wang, G., et al. (2007). Comparative pharmacokinetics of baicalin after oral administration of pure baicalin, Radix scutellariae extract and Huang-Lian-Jie-Du-Tang to rats. J. Ethnopharmacol. 110 (3), 412-418. doi: 10.1016/j.jep.2006.09.036

Lu, Q. Y., Zhang, L., Moro, A., Chen, M. C., Harris, D. M., Eibl, G., et al. (2012). Detection of baicalin metabolites baicalein and oroxylin-a in mouse pancreas and pancreatic xenografts. Pancreas 41 (4), 571-576. doi: 10.1097/ MPA.0b013e318232e130

Luan, Y., Sun, C., Wang, J., Jiang, W., Xin, Q., Zhang, Z., et al. (2019). Baicalin attenuates myocardial ischemia-reperfusion injury through Akt/NF- $\kappa B$ pathway. J. Cell. Biochem. 120 (3), 3212-3219. doi: 10.1002/jcb.27587

Lv, P., Zhang, F., Yin, Y. J., Wang, Y. C., Gao, M., Xie, X. L., et al. (2016). SM22 $\alpha$ inhibits lamellipodium formation and migration via Ras-Arp2/3 signaling in synthetic VSMCs. American journal of physiology. Cell Physiol. 311 (5), C758C767. doi: 10.1152/ajpcell.00033.2016

Ma, S., Zhao, M., Liu, H., Wang, L., and Zhang, X. (2012). Pharmacokinetic effects of baicalin on cerebral ischemia-reperfusion after iv administration in rats. Chin. Herbal Med. 4 (1), 53-57. doi: 10.3969/j.issn.1674-6384.2012.01.008

Ma, L., Liu, H., Xie, Z., Yang, S., Xu, W., Hou, J., et al. (2014). Ginsenoside Rb3 protects cardiomyocytes against ischemia-reperfusion injury via the inhibition of JNK-mediated NF-KB pathway: a mouse cardiomyocyte model. PloS One 9 (8), e103628. doi: 10.1371/journal.pone.0103628

Marson, A., Kretschmer, K., Frampton, G. M., Jacobsen, E. S., Polansky, J. K., MacIsaac, K. D., et al. (2007). Foxp3 occupancy and regulation of key target genes during T-cell stimulation. Nature 445 (7130), 931-935. doi: 10.1038/ nature 05478

Mehta, P. K., and Griendling, K. K. (2007). Angiotensin II cell signaling: physiological and pathological effects in the cardiovascular system. American journal of physiology. Cell Physiol. 292 (1), C82-C97. doi: 10.1152/ajpcell.00287.2006

Moore, K. J., and Tabas, I. (2011). Macrophages in the pathogenesis of atherosclerosis. Cell 145 (3), 341-355. doi: 10.1016/j.cell.2011.04.005

Nègre-Salvayre, A., Augé, N., Camaré, C., Bacchetti, T., Ferretti, G., and Salvayre, R. (2017). Dual signaling evoked by oxidized LDLs in vascular cells. Free Radical Biol. Med. 106, 118-133. doi: 10.1016/j.freeradbiomed.2017.02.006

Niehrs, C. (2006). Function and biological roles of the Dickkopf family of Wnt modulators. Oncogene 25 (57), 7469-7481. doi: 10.1038/sj.onc.1210054

Noh, K., Kang, Y., Nepal, M. R., Jeong, K. S., Oh, D. G., Kang, M. J., et al. (2016). Role of Intestinal Microbiota in Baicalin-Induced Drug Interaction and Its Pharmacokinetics. Mol. (Basel Switzerland) 21 (3), 337. doi: 10.3390/ molecules 21030337

Ohashi, R., Mu, H., Wang, X., Yao, Q., and Chen, C. (2005). Reverse cholesterol transport and cholesterol efflux in atherosclerosis. QJM Monthly J. Assoc. Physicians 98 (12), 845-856. doi: 10.1093/qjmed/hci136

Olofsson, S. O., and Borèn, J. (2005). Apolipoprotein B: a clinically important apolipoprotein which assembles atherogenic lipoproteins and promotes the development of atherosclerosis. J. Internal Med. 258 (5), 395-410. doi: 10.1111/ j.1365-2796.2005.01556.x
Olsvik, P. A., Kristensen, T., Waagbø, R., Rosseland, B. O., Tollefsen, K. E., Baeverfjord, G., et al. (2005). mRNA expression of antioxidant enzymes (SOD, CAT and GSH-Px) and lipid peroxidative stress in liver of Atlantic salmon (Salmo salar) exposed to hyperoxic water during smoltification. Comp. Biochem. Physiol. Toxicol. Pharmacol. CBP 141 (3), 314-323. doi: 10.1016/ j.cbpc.2005.07.009

Packard, R. R., Lichtman, A. H., and Libby, P. (2009). Innate and adaptive immunity in atherosclerosis. Semin. Immunopathol. 31 (1), 5-22. doi: 10.1007/s00281-009-0153-8

Pang, J. J., Barton, L. A., Chen, Y. G., and Ren, J. (2015). Mitochondrial aldehyde dehydrogenase in myocardial ischemia-reperfusion injury: from bench to bedside. Sheng Li Xue Bao [Acta Physiol. Sinica] 67 (6), 535-544.

Parati, G. (2015). Antihypertensive therapy in 2014: Linking pathophysiology to antihypertensive treatment. Nat. Rev. Cardiol. 12 (2), 77-79. doi: 10.1038/ nrcardio.2014.221

Pasupathy, S., Air, T., Dreyer, R. P., Tavella, R., and Beltrame, J. F. (2015). Systematic review of patients presenting with suspected myocardial infarction and nonobstructive coronary arteries. Circulation 131 (10), 861-870. doi: 10.1161/CIRCULATIONAHA.114.011201

Peifer, M., and Polakis, P. (2000). Wnt signaling in oncogenesis and embryogenesis-a look outside the nucleus. Sci. (N. Y. N. Y.) 287 (5458), 1606-1609. doi: 10.1126/science.287.5458.1606

Peluso, I., Morabito, G., Urban, L., Ioannone, F., and Serafini, M. (2012). Oxidative stress in atherosclerosis development: the central role of LDL and oxidative burst. Endocrine Metab. Immune Disord. Drug Targets 12 (4), 351-360. doi: $10.2174 / 187153012803832602$

Petrich, B. G., and Wang, Y. (2004). Stress-activated MAP kinases in cardiac remodeling and heart failure; new insights from transgenic studies. Trends Cardiovasc. Med. 14 (2), 50-55. doi: 10.1016/j.tcm.2003.11.002

Pizzino, G., Irrera, N., Cucinotta, M., Pallio, G., Mannino, F., Arcoraci, V., et al. (2017). Oxidative Stress: Harms and Benefits for Human Health. Oxid. Med. Cell. Longevity 2017, 8416763. doi: 10.1155/2017/8416763

Potekhina, A. V., Pylaeva, E., Provatorov, S., Ruleva, N., Masenko, V., Noeva, E., et al. (2015). Treg/Th17 balance in stable CAD patients with different stages of coronary atherosclerosis. Atherosclerosis 238 (1), 17-21. doi: 10.1016/ j.atherosclerosis.2014.10.088

Prabhu, S. D., and Frangogiannis, N. G. (2016). The Biological Basis for Cardiac Repair After Myocardial Infarction: From Inflammation to Fibrosis. Circ. Res. 119 (1), 91-112. doi: 10.1161/CIRCRESAHA.116.303577

Prech, M., Marszałek, A., Schröder, J., Filas, V., Lesiak, M., Jemielity, M., et al. (2010). Apoptosis as a mechanism for the elimination of cardiomyocytes after acute myocardial infarction. Am. J. Cardiol. 105 (9), 1240-1245. doi: 10.1016/ j.amjcard.2009.12.039

Qiu, L., Chen, J., Lin, J., Wo, D., Chu, J., and Peng, J. (2017). Baicalin alleviates $\mathrm{H} 2 \mathrm{O} 2$-induced injury of $\mathrm{H} 9 \mathrm{c} 2$ cardiomyocytes through suppression of the Wnt/ $\beta$-catenin signaling pathway. Mol. Med. Rep. 16 (6), 9251-9255. doi: $10.3892 / \mathrm{mmr} .2017 .7748$

Reimer, K. A., Vander Heide, R. S., and Richard, V. J. (1993). Reperfusion in acute myocardial infarction: effect of timing and modulating factors in experimental models. Am. J. Cardiol. 72 (19), 13G-21G. doi: 10.1016/0002-9149(93)90102-i

Reiner, Ž., Catapano, A. L., De Backer, G., Graham, I., Taskinen, M. R., Wiklund, O., et al. (2011). ESC/EAS guidelines for the management of dyslipidaemias: the task force for the management of dyslipidaemias of the European Society of Cardiology (ESC) and the European Atherosclerosis Society (EAS). Eur. Heart J. 32, 1769-1818. doi: 10.1093/eurheartj/ehr158

Ross, R. (1999). Atherosclerosis-an inflammatory disease. New Engl. J. Med. 340 (2), 115-126. doi: 10.1056/NEJM199901143400207

Sampaio, W. O., Souza dos Santos, R. A., Faria-Silva, R., da Mata Machado, L. T., Schiffrin, E. L., and Touyz, R. M. (2007). Angiotensin-(1-7) through receptor Mas mediates endothelial nitric oxide synthase activation via Akt-dependent pathways. Hypertension (Dallas Tex. 1979) 49 (1), 185-192. doi: 10.1161/ 01.HYP.0000251865.35728.2f

Santisteban, M. M., Qi, Y., Zubcevic, J., Kim, S., Yang, T., Shenoy, V., et al. (2017). Hypertension-Linked Pathophysiological Alterations in the Gut. Circ. Res. 120 (2), 312-323. doi: 10.1161/CIRCRESAHA.116.309006

Sen, M., and Ghosh, G. (2008). Transcriptional outcome of Wnt-Frizzled signal transduction in inflammation: evolving concepts. J. Immunol. (Baltimore Md.: 1950) 181 (7), 4441-4445. doi: 10.4049/jimmunol.181.7.4441 
Shang, X., He, X., He, X., Li, M., Zhang, R., Fan, P., et al. (2010). The genus Scutellaria an ethnopharmacological and phytochemical review. J. Ethnopharmacol. 128 (2), 279-313. doi: 10.1016/j.jep.2010.01.006

Shen, J., Yang, M., Ju, D., Jiang, H., Zheng, J. P., Xu, Z., et al. (2010). Disruption of SM22 promotes inflammation after artery injury via nuclear factor kappaB activation. Circ. Res. 106 (8), 1351-1362. doi: 10.1161/CIRCRESAHA.109. 213900

Shimada, K. (2009). Immune system and atherosclerotic disease: heterogeneity of leukocyte subsets participating in the pathogenesis of atherosclerosis. Circ. J. 73 (6), 994-1001. doi: 10.1253/circj.cj-09-0277

Shu, Y. N., Zhang, F., Bi, W., Dong, L. H., Zhang, D. D., Chen, R., et al. (2015).

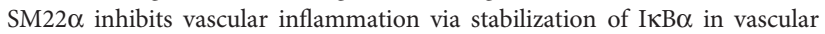
smooth muscle cells. J. Mol. Cell. Cardiol. 84, 191-199. doi: 10.1016/ j.yjmcc.2015.04.020

Song, Y., and Yang, J. M. (2017). Role of interleukin (IL)-17 and T-helper (Th)17 cells in cancer. Biochem. Biophys. Res. Commun. 493 (1), 1-8. doi: 10.1016/ j.bbrc.2017.08.109

Srinivas, N. R. (2010). Baicalin, an emerging multi-therapeutic agent: pharmacodynamics, pharmacokinetics, and considerations from drug development perspectives. Xenobiot. Fate Foreign Compounds Biol. Syst. 40 (5), 357-367. doi: 10.3109/00498251003663724

Steinman, R. M., and Hemmi, H. (2006). Dendritic cells: translating innate to adaptive immunity. Curr. Topics Microbiol. Immunol. 311, 17-58. doi: 10.1007/ 3-540-32636-7_2

Steinman, R. M., Adams, J. C., and Cohn, Z. A. (1975). Identification of a novel cell type in peripheral lymphoid organs of mice. IV. Identification and distribution in mouse spleen. J. Exp. Med. 141 (4), 804-820.

Sun, A., Cheng, Y., Zhang, Y., Zhang, Q., Wang, S., Tian, S., et al. (2014). Aldehyde dehydrogenase 2 ameliorates doxorubicin-induced myocardial dysfunction through detoxification of 4-HNE and suppression of autophagy. J. Mol. Cell. Cardiol. 71, 92-104. doi: 10.1016/j.yjmcc.2014.01.002

Sun, S. J., Wu, X. P., Song, H. L., and Li, G. Q. (2015). Baicalin ameliorates isoproterenol-induced acute myocardial infarction through iNOS, inflammation, oxidative stress and P38MAPK pathway in rat. Int. J. Clin. Exp. Med. 8 (12), 22063-22072.

Tan, H. Y., Wang, N., Li, S., Hong, M., Wang, X., and Feng, Y. (2016). The Reactive Oxygen Species in Macrophage Polarization: Reflecting Its Dual Role in Progression and Treatment of Human Diseases. Oxid. Med. Cell. Longevity 2016, 2795090. doi: 10.1155/2016/2795090

Tang, Y., Zhu, H., Zhang, Y., and Huang, C. (2006). Determination of human plasma protein binding of baicalin by ultrafiltration and high-performance liquid chromatography. Biomed. Chromatography: BMC 20 (10), 1116-1119. doi: 10.1002/bmc.655

Timmis, A., Townsend, N., Gale, C., Grobbee, R., Maniadakis, N., and Flather, M. (2018). European Society of Cardiology: Cardiovascular Disease Statistics 2017. Eur. Heart J. 39 (7), 508-579. doi: 10.1093/eurheartj/ehx628

Tong, F., and Zhou, X. (2017). The Nrf2/HO-1 Pathway Mediates the Antagonist Effect of L-Arginine On Renal Ischemia/Reperfusion Injury in Rats. Kidney Blood Pressure Res. 42 (3), 519-529. doi: 10.1159/000480362

Tousoulis, D., Psaltopoulou, T., Androulakis, E., Papageorgiou, N., Papaioannou, S., Oikonomou, E., et al. (2015). Oxidative stress and early atherosclerosis: novel antioxidant treatment. Cardiovasc. Drugs Ther. 29 (1), 75-88. doi: 10.1007/ s10557-014-6562-5

Trigatti, B., Covey, S., and Rizvi, A. (2004). Scavenger receptor class B type I in high-density lipoprotein metabolism, atherosclerosis and heart disease: lessons from gene-targeted mice. Biochem. Soc. Trans. 32 (Pt 1), 116-120. doi: 10.1042/ bst0320116

Tse, K., Tse, H., Sidney, J., Sette, A., and Ley, K. (2013). T cells in atherosclerosis. Int. Immunol. 25 (11), 615-622. doi: 10.1093/intimm/dxt043

Ueland, T., Otterdal, K., Lekva, T., Halvorsen, B., Gabrielsen, A., Sandberg, W. J., et al. (2009). Dickkopf-1 enhances inflammatory interaction between platelets and endothelial cells and shows increased expression in atherosclerosis. Arteriosclerosis Thrombosis Vasc. Biol. 29 (8), 1228-1234. doi: 10.1161/ ATVBAHA.109.189761

Van den Akker, F., de Jager, S. C., and Sluijter, J. P. (2013). Mesenchymal stem cell therapy for cardiac inflammation: immunomodulatory properties and the influence of toll-like receptors. Mediators Inflammation 2013, 181020. doi: $10.1155 / 2013 / 181020$
Vander Heide, R. S., and Steenbergen, C. (2013). Cardioprotection and myocardial reperfusion: pitfalls to clinical application. Circ. Res. 113 (4), 464-477. doi: 10.1161/CIRCRESAHA.113.300765

Vandervelde, S., van Amerongen, M. J., Tio, R. A., Petersen, A. H., van Luyn, M. J., and Harmsen, M. C. (2006). Increased inflammatory response and neovascularization in reperfused vs. non-reperfused murine myocardial infarction. Cardiovasc. Pathol. 15 (2), 83-90. doi: 10.1016/j.carpath. 2005.10.006

Virani, S. S., Alonso, A., Benjamin, E. J., Bittencourt, M. S., Callaway, C. W., Carson, A. P., et al. (2020). American Heart Association Council on Epidemiology and Prevention Statistics Committee and Stroke Statistics Subcommittee Heart Disease and Stroke Statistics-2020 Update: A Report From the American Heart Association. Circulation 141 (9), e139-e596. doi: 10.1161/CIR.0000000000000757

Wang, Y., Yang, J., Li, X., and Wang, J. (2012). The metabolism of baicalin in rat and the biological activities of the metabolites. Evidence-Based Complementary Altern. Med. eCAM 2012, 404529. doi: 10.1155/2012/404529

Wang, X., He, F., Liao, Y., Song, X., Zhang, M., Qu, L., et al. (2013). Baicalin pretreatment protects against myocardial ischemia/reperfusion injury by inhibiting mitochondrial damage-mediated apoptosis. Int. J. Cardiol. 168 (4), 4343-4345. doi: 10.1016/j.ijcard.2013.05.077

Wang, B., Liao, P. P., Liu, L. H., Fang, X., Li, W., and Guan, S. M. (2016). Baicalin and geniposide inhibit the development of atherosclerosis by increasing Wnt1 and inhibiting dickkopf-related protein-1 expression. J. Geriatr. Cardiol.: JGC 13 (10), 846-854. doi: 10.11909/j.issn.1671-5411.2016.10.013

Wang, L., Li, Y., Lin, S., Pu, Z., Li, H., and Tang, Z. (2018). Protective Effects of Baicalin on Experimental Myocardial Infarction in Rats. Braz. J. Cardiovasc. Surg. 33 (4), 384-390. doi: 10.21470/1678-9741-2018-0059

Wang, Z. L., Wang, S., Kuang, Y., Hu, Z. M., Qiao, X., and Ye, M. (2018). A comprehensive review on phytochemistry, pharmacology, and flavonoid biosynthesis of Scutellaria baicalensis. Pharm. Biol. 56 (1), 465-484. doi: 10.1080/13880209.2018.1492620

Weber, C., and Noels, H. (2011). Atherosclerosis: current pathogenesis and therapeutic options. Nat. Med. 17 (11), 1410-1422. doi: 10.1038/nm.2538

Wei, Y., Guo, J., Zheng, X., Wu, J., Zhou, Y., Yu, Y., et al. (2014). Preparation, pharmacokinetis and biodistribution of baicalin-loaded liposomes. Int. J. Nanomed. 9, 3623-3630. doi: 10.2147/IJN.S66312

Wei, X., Zhu, X., Hu, N., Zhang, X., Sun, T., Xu, J., et al. (2015). Baicalin attenuates angiotensin II-induced endothelial dysfunction. Biochem. Biophys. Res. Commun. 465 (1), 101-107. doi: 10.1016/j.bbrc.2015.07.138

Wei, Y., Pi, C., Yang, G., Xiong, X., Lan, Y., Yang, H., et al. (2016). LC-UV Determination of Baicalin in Rabbit Plasma and Tissues for Application in Pharmacokinetics and Tissue Distribution Studies of Baicalin after Intravenous Administration of Liposomal and Injectable Formulations. Mol. (Basel Switzerland) 21 (4), 444. doi: 10.3390/molecules21040444

Wencker, D., Chandra, M., Nguyen, K., Miao, W., Garantziotis, S., Factor, S. M., et al. (2003). A mechanistic role for cardiac myocyte apoptosis in heart failure. J. Clin. Invest. 111 (10), 1497-1504. doi: 10.1172/JCI17664

Wolf, D., and Ley, K. (2019). Immunity and Inflammation in Atherosclerosis. Circ. Res. 124 (2), 315-327. doi: 10.1161/CIRCRESAHA.118.313591

Wu, Y., Wang, F., Fan, L., Zhang, W., Wang, T., Du, Y., et al. (2018). Baicalin alleviates atherosclerosis by relieving oxidative stress and inflammatory responses via inactivating the NF- $\mathrm{KB}$ and p38 MAPK signaling pathways. Biomed. Pharmacother. Biomed. Pharmacother. 97, 1673-1679. doi: 10.1016/ j.biopha.2017.12.024

Wu, D., Ding, L., Tang, X., Wang, W., Chen, Y., and Zhang, T. (2019). Baicalin Protects Against Hypertension-Associated Intestinal Barrier Impairment in Part Through Enhanced Microbial Production of Short-Chain Fatty Acids. Front. Pharmacol. 10, 1271. doi: 10.3389/fphar.2019.01271

Wu, J., Nakashima, S., Nakamura, S., and Matsuda, H. (2020). Effects of Sanoshashinto on left ventricular hypertrophy and gut microbiota in spontaneously hypertensive rats. J. Nat. Med. 74 (2), 482-486. doi: 10.1007/ s11418-020-01387-9

Xi, Y., Wu, M., Li, H., Dong, S., Luo, E., Gu, M., et al. (2015). Baicalin Attenuates High Fat Diet-Induced Obesity and Liver Dysfunction: Dose-Response and

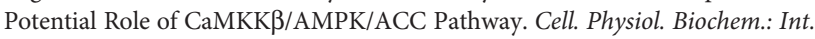
J. Exp. Cell. Physiol. Biochem. Pharmacol. 35 (6), 2349-2359. doi: 10.1159/ 000374037 
Xiao, Y., Ye, J., Zhou, Y., Huang, J., Liu, X., Huang, B., et al. (2018). Baicalin inhibits pressure overload-induced cardiac fibrosis through regulating AMPK/ TGF- $\beta /$ Smads signaling pathway. Arch. Biochem. Biophysics 640, 37-46. doi: 10.1016/j.abb.2018.01.006

Xing, J., Chen, X., and Zhong, D. (2005). Absorption and enterohepatic circulation of baicalin in rats. Life Sci. 78 (2), 140-146. doi: 10.1016/j.lfs.2005.04.072

Yancy, C. W., Jessup, M., Bozkurt, B., Butler, J., Casey, D. E. C.OMMAJ.R.X.X.X, Drazner, M. H., et al. (2013). 2013 ACCF/AHA guideline for the management of heart failure: executive summary: a report of the American College of Cardiology Foundation/American Heart Association Task Force on practice guidelines. Circulation 128 (16), 1810-1852. doi: 10.1161/CIR.0b013 e31829e8807

Yang, J., Yang, X., Chu, Y., and Li, M. (2011). Identification of Baicalin as an immunoregulatory compound by controlling $\mathrm{T}(\mathrm{H}) 17$ cell differentiation. PloS One 6 (2), e17164. doi: 10.1371/journal.pone.0017164

Yang, J., Yang, X., and Li, M. (2012). Baicalin, a natural compound, promotes regulatory $\mathrm{T}$ cell differentiation. BMC Complementary Altern. Med. 12, 64. doi: $10.1186 / 1472-6882-12-64$

Yang, J., Fan, Z., Yang, J., Ding, J., Yang, C., and Chen, L. (2016). microRNA-22 attenuates myocardial ischemia-reperfusion injury via an anti-inflammatory mechanism in rats. Exp. Ther. Med. 12 (5), 3249-3255. doi: 10.3892/ etm.2016.3777

Ye, L., Tao, Y., Wang, Y., Feng, T., and Li, H. (2015). The effects of baicalin on the TLR2/4 signaling pathway in the peripheral blood mononuclear cells of a lipopolysaccharide-induced rat fever model. Int. Immunopharmacol. 25 (1), 106-111. doi: 10.1016/j.intimp.2014.12.028

Yu, X. H., Fu, Y. C., Zhang, D. W., Yin, K., and Tang, C. K. (2013). Foam cells in atherosclerosis. Clin. Chim. Acta; Int. J. Clin. Chem. 424, 245-252. doi: 10.1016/j.cca.2013.06.006

Yu, R., Lv, Y., Wang, J., Pan, N., Zhang, R., Wang, X., et al. (2016). Baicalin promotes cholesterol efflux by regulating the expression of SR-BI in macrophages. Exp. Ther. Med. 12 (6), 4113-4120. doi: 10.3892/etm.2016.3884

Yu, H., Chen, B., and Ren, Q. (2019). Baicalin relieves hypoxia-aroused H9c2 cell apoptosis by activating Nrf2/HO-1-mediated HIF1 $\alpha / \mathrm{BNIP} 3$ pathway. Artif. Cells Nanomed. Biotechnol. 47 (1), 3657-3663. doi: 10.1080/21691401.2019.1657879

Yue, T. L., Wang, C., Gu, J. L., Ma, X. L., Kumar, S., Lee, J. C., et al. (2000). Inhibition of extracellular signal-regulated kinase enhances Ischemia/ Reoxygenation-induced apoptosis in cultured cardiac myocytes and exaggerates reperfusion injury in isolated perfused heart. Circ. Res. 86 (6), 692-699. doi: 10.1161/01.res.86.6.692

Yue, P. F., Li, Y., Wan, J., Wang, Y., Yang, M., Zhu, W. F., et al. (2013). Process optimization and evaluation of novel baicalin solid nanocrystals. Int. J. Nanomed. 8, 2961-2973. doi: 10.2147/IJN.S44924

Zeng, X., Li, J., and Li, Z. (2015). Ginsenoside Rd mitigates myocardial ischemiareperfusion injury via $\mathrm{Nrf} 2 / \mathrm{HO}-1$ signaling pathway. Int. J. Clin. Exp. Med. 8 (8), 14497-14504.

Zhang, Z., Deb, A., Zhang, Z., Pachori, A., He, W., Guo, J., et al. (2009). Secreted frizzled related protein 2 protects cells from apoptosis by blocking the effect of canonical Wnt3a. J. Mol. Cell. Cardiol. 46 (3), 370-377. doi: 10.1016/ j.yjmcc.2008.11.016

Zhang, P., Xu, D., Wang, S., Fu, H., Wang, K., Zou, Y., et al. (2011). Inhibition of aldehyde dehydrogenase 2 activity enhances antimycin-induced rat cardiomyocytes apoptosis through activation of MAPK signaling pathway. Biomed. Pharmacother. Biomed. Pharmacother. 65 (8), 590-593. doi: 10.1016/ j.biopha.2009.12.001

Zhang, H., Yang, X., Zhao, L., Jiao, Y., Liu, J., and Zhai, G. (2016). In vitro and in vivo study of Baicalin-loaded mixed micelles for oral delivery. Drug Deliv. 23 (6), 1933-1939. doi: 10.3109/10717544.2015.1008705
Zhang, J., Zhang, S., Teng, S., and Zhai, L. (2016). An LC-MS/MS method for simultaneous determination of four flavonoids from Semen Oroxyli in rat plasma and its application to a pharmacokinetic study. J. Chromatography B. Analyt. Technol. Biomed. Life Sci. 1020, 96-102. doi: 10.1016/j.jchromb. 2016.03.030

Zhang, R., Cui, Y., Wang, Y., Tian, X., Zheng, L., Cong, H., et al. (2017). CatecholO-Methyltransferase and UDP-Glucuronosyltransferases in the Metabolism of Baicalein in Different Species. Eur. J. Drug Metab. Pharmacokinet. 42 (6), 981992. doi: 10.1007/s13318-017-0419-9

Zhang, Y., Liao, P., Zhu, M., Li, W., Hu, D., Guan, S., et al. (2017). Baicalin Attenuates Cardiac Dysfunction and Myocardial Remodeling in a Chronic Pressure-Overload Mice Model. Cell. Physiol. Biochem.: Int. J. Exp. Cell. Physiol. Biochem. Pharmacol. 41 (3), 849-864. doi: 10.1159/000459708

Zhang, Z. (1974). "Around $200 \mathrm{AD}$ and republished in 1974," in ShanghanLun (On cold damage) (Beijing: People's Medical Publishing House), p 27.

Zhao, Y., Kong, H., Sun, Y., Feng, H., Zhang, Y., Su, X., et al. (2014). Assessment of baicalin in mouse blood by monoclonal antibody-based icELISA. Biomed. Chromatogr. BMC 28 (12), 1864-1868. doi: 10.1002/bmc.3235

Zhao, Q., Chen, X. Y., and Martin, C. (2016). Scutellaria baicalensis, the golden herb from the garden of Chinese medicinal plants. Sci. Bull. 61 (18), 13911398. doi: 10.1007/s11434-016-1136-5

Zhao, D., Liu, J., Wang, M., Zhang, X., and Zhou, M. (2019). Epidemiology of cardiovascular disease in China: current features and implications. Nat. Rev. Cardiol. 16 (4), 203-212. doi: 10.1038/s41569-018-0119-4

Zhao, T., Tang, H., Xie, L., Zheng, Y., Ma, Z., Sun, Q., et al. (2019). Scutellaria baicalensis Georgi. (Lamiaceae): a review of its traditional uses, botany, phytochemistry, pharmacology and toxicology. J. Pharm. Pharmacol. 71 (9), 1353-1369. doi: 10.1111/jphp.13129

Zhu, Q., Li, X. X., Wang, W., Hu, J., Li, P. L., Conley, S., et al. (2016). Mesenchymal stem cell transplantation inhibited high salt-induced activation of the NLRP3 inflammasome in the renal medulla in Dahl S rats. American journal of physiology. Renal Physiol. 310 (7), F621-F627. doi: 10.1152/ajprenal. 00344.2015

Zhu, W., Jin, Z., Yu, J., Liang, J., Yang, Q., Li, F., et al. (2016). Baicalin ameliorates experimental inflammatory bowel disease through polarization of macrophages to an M2 phenotype. Int. Immunopharmacol. 35, 119-126. doi: 10.1016/j.intimp.2016.03.030

Zhu, Y., Xian, X., Wang, Z., Bi, Y., Chen, Q., Han, X., et al. (2018). Research Progress on the Relationship between Atherosclerosis and Inflammation. Biomolecules 8 (3), 80. doi: 10.3390/biom 8030080

Zidar, N., Jera, J., Maja, J., and Dusan, S. (2007). Caspases in myocardial infarction. Adv. Clin. Chem. 44, 1-33. doi: 10.1016/s0065-2423(07)44001-x

Zizzo, G., Hilliard, B. A., Monestier, M., and Cohen, P. L. (2012). Efficient clearance of early apoptotic cells by human macrophages requires M2c polarization and MerTK induction. J. Immunol. (Baltimore Md. 1950) 189 (7), 3508-3520. doi: 10.4049/jimmunol.1200662

Conflict of Interest: The authors declare that the research was conducted in the absence of any commercial or financial relationships that could be construed as a potential conflict of interest.

Copyright (C) 2020 Xin, Gao, Lin, Qu, Shang, Wang, Lu and Cui. This is an openaccess article distributed under the terms of the Creative Commons Attribution License (CC BY). The use, distribution or reproduction in other forums is permitted, provided the original author(s) and the copyright owner(s) are credited and that the original publication in this journal is cited, in accordance with accepted academic practice. No use, distribution or reproduction is permitted which does not comply with these terms. 